Draft Version SEPtember 26, 2017

Preprint typeset using LATEX style emulateapj v. 12/16/11

\title{
PROBING THE SPINNING OF THE MASSIVE BLACK HOLE IN THE GALACTIC CENTER VIA PULSAR TIMING: A FULL RELATIVISTIC TREATMENT
}

\author{
Fupeng Zhang ${ }^{1, \dagger}$, Prasenjit Saha ${ }^{2}$ \\ ${ }^{1}$ School of Physics and Astronomy, Sun Yat-Sen University, Guangzhou 510275, China; ${ }^{\dagger}$ zhangfp7@mail.sysu.edu.cn; \\ 2 Physik-Institut, University of Zürich, Switzerland, psaha@physik.uzh.ch \\ Draft version September 26, 2017
}

\section{ABSTRACT}

\begin{abstract}
Pulsars around the Massive Black Hole (MBH) in the Galactic Center (GC) are expected to be revealed by the incoming facilities (e.g., the Square Kilometre Array). Under a full relativistic framework with the pulsar approximated as a test particle, we investigate the constraints on the spinning of the MBH by monitoring the timing of surrounding pulsars. For GC pulsars orbiting closely around the MBH (e.g., $\lesssim 1000 \mathrm{AU}$ ), we find that full relativistic treatment in modeling accurately their timing signals can be necessary, as the relativistic signals are orders of magnitude larger than the time of arrival measurement accuracies. Although usually there are near-degeneracies among MBH spin parameters, the constraints on the spinning of the $\mathrm{MBH}$ are still very tight. By continuously monitoring a normal pulsar in orbits with a period of $\sim 2.6 \mathrm{yr}$ and an eccentricity of $0.3-0.9$ under timing precision of $1-5 \mathrm{~ms}$, within $\sim 8 \mathrm{yr}$ the spin magnitude and the orientations of the GC MBH can be constrained with $2 \sigma$ error of $10^{-3}-10^{-2}$ and $10^{-1}-10^{\circ}$, respectively. Even for pulsars in orbits similar to the detected star S2/S0-2 or S0-102, we find that the spinning of the MBH can still be constrained within $4-8 \mathrm{yr}$, with the most significant constraints provided near the pericenter passage. If the proper motion of the pulsars with astrometric accuracy of $10 \mu$ as can also be collected along with the timing measurement, then the position, velocity, mass and the distance to the Solar System of the MBH can be constrained about $\sim 10 \mu \mathrm{as}, \sim 1 \mu \mathrm{as} / \mathrm{yr}, \sim 10 M_{\odot}$ and $\sim 1 \mathrm{pc}$, respectively.

Keywords: black hole physics - Galaxy: center - Galaxy: nucleus - gravitation - relativistic processes - pulsars: general
\end{abstract}

\section{INTRODUCTION}

Due to their tremendous rotation stability, pulsars are believed to be one of the best probes in testing gravity theories in various astrophysical environments (for reviews, see e.g., Stairs 2003; Will 2014; Lorimer 2008). Binary pulsars have provided clean tests of various general relativistic (GR) effects, including the decay of the orbital period by gravitational wave radiation (Taylor 1994; Kramer et al. 2006). Such systems present cases of comparatively weak gravitational fields, i.e., with $G M / r c^{2}$ of $10^{-5}-10^{-7}$, where $M$ is the mass of the system and $r$ is the distance between the two components. On the other hand, the so-called "Sstars" near the Galactic Center (GC) Ghez et al. 2008; Gillessen et al. 2009, 2017) delve into $\sim G M / r c^{2} \simeq 10^{-3}$ (see, for example, Figure 1 of Angélil et al. 2010). In this region, $M \simeq 4 \times 10^{6} M_{\odot}$ is a massive black hole $(\mathrm{MBH})$. If pulsars orbiting quite close to the MBH could be found, the precise tracing of time of arrival (TOA) of their pulses can be used for probing the Kerr spacetime near a black hole.

The existence of pulsars close to the GC MBH are inferred by the discovery of hundreds of young and massive stars within the inner parsec of GC (e.g., Paumard et al. 2006; Lu et al. 2013). Some of those massive stars (e.g., $\left.\gtrsim 9 M_{\odot}\right)$ can leave neutron star remnants at the end of their lifetime through supernova explosions. The number of these pulsars is expected to be about 100 within the orbital period of $100 \mathrm{yr}$ (e.g. Zhang et al. 2014; Pfahl \& Loeb 2004; Chennamangalam \& Lorimer 2014). The innermost one of them could be in an orbit as tight as about $\sim 100-500$ AU from the MBH (Zhang et al. 2014). The existence of a population of normal pulsars in the GC has also been strongly suggested by the magnetar recently revealed in this region, as magnetars are rare pulsars (e.g., Rea et al. 2013; Eatough et al. 2013).

The severe broadening of the pulse profile due to the hyper-strong radio-wave scattering by the interstellar media in GC imposes difficulties in revealing the existence of these objects. The search of pulsars in GC has to be performed in high radio frequencies (e.g., usually $\gtrsim 9 \mathrm{GHz}$ ) (Cordes \& Lazio 1997; Pfahl \& Loeb 2004). Although a number of GC pulsar searches have been performed, no normal pulsars have been detected within the inner parsec so far (e.g., Deneva et al. 2009; Macquart et al. 2010; Bates et al. 2011). Future facilities, e.g., Square Kilometre Array (SKA), may be able to reveal a number of normal pulsars in this region, due to its very large collection area, offering prospects of testing general relativity by their timing observations (e.g., Shao et al. 2015; Eatough et al. 2015).

Pulsar binaries with the GC-MBH would have some important difference from known pulsar binaries.

- First, the orbital periods around the GC-MBH would be years or decades, compared to hours to weeks for stellar binaries with white dwarfs or neutron stars. The same applies to $\mathrm{S}$ stars. As a result, orbital precession or indeed any orbit-averaged quantity is not the most useful variable. The earlier literature on orbits around the GC-MBH (whether S stars or pulsars) tended to focus on precession (e.g., Jaroszynski 1998; Rubilar \& Eckart 2001; 
Pfahl \& Loeb 2004; Will 2008; Merritt et al. 2011; Liu et al. 2012; (Psaltis et al. 2016). More recent literature has emphasized relativistic effects that appear within a few orbits, especially near pericenter passages (Angélil \& Saha 2010; Angélil et al. 2010; Zhang et al. 2015; Yu et al. 2016).

- Second, in stellar-mass binaries it is essential to consider the gravity of both bodies, which is typically done through a post-Newtonian treatment (Blandford \& Teukolskv 1976; Damour \& Deruelle 1986; Hobbs et al. 2006). For a pulsar-MBH binary, the mass ratio is much smaller $\left(\sim 10^{-7}-10^{-6}\right)$ and the pulsar can be approximated by a test particle moving in a Kerr metric (see Section 4.4 for the consequences of such an approximation).

- There are, however, Newtonian dynamical perturbations from all the other masses in the GC region (Merritt et al. 2011; Zhang \& Iorio 2017). How to remove out this Newtonian "foreground" remains an unsolved problem. A possible filtering strategy using wavelets is suggested by Angélil \& Saha (2014).

This work studies a pulsar in a Kerr metric. The full relativistic framework developed previously (Zhang et al. 2015, hereafter ZLY15) to simulate orbits and redshifts is modified to compute pulsar TOAs instead. Note that although we describe the method as "full relativistic treatment" of the pulsar's motion, we have neglected the mass of the pulsar (For the difference if the pulsar's mass is not ignored see also Section 4.4.) and the orbital decay due to the gravitational wave radiation. By performing a large number of Markov Chain Monte Carlo (MCMC) simulations, we investigate the constraints on the spinning of the $\mathrm{MBH}$ for pulsars in various orbits and under different timing accuracies. Meanwhile, the proper motion of the pulsars measured by radio astrometry could also be quite significant as they are very close to the $\mathrm{MBH}$. Here we investigate the possible benefits of including the proper motion measurements of the pulsars, e.g., the additional constraints on the mass, distance and proper motion of the $\mathrm{MBH}$.

This paper is organized as follows: In Section 2 we introduce the details of the numerical integration of the motion of pulsar and the pulse trajectory from the pulsar to the observer under the Kerr metric. By such a full relativistic method, we derive the observables, i.e., the TOA and the proper motion of the target pulsar. We have extended the previous relativistic framework in ZLY15 by including the motion of the MBH itself in the simulation. We investigate the spin-induced GR effects in pulsar timing and proper motion for some hypothetical pulsars in Section 3. This section also compares with orbit-averaged post-Newtonian theory for pulsar binaries, and shows that such approximations could deviate from the real evolution of the orbital precession in the full GR case, with the differences that could be apparent by timing measurements of pulsars in GC (see also Section 3.4.1 or Figure (4). In Section 4 we perform a large number of Markov chain Monte Carlo simulations to investigate the constraints on the parameters of the $\mathrm{MBH}$, including the spin, mass, proper motion and the GC distance. The discussion and the conclusions are shown in Sections 5 and 6 , respectively.

\section{NUMERICAL METHODS}

In this work, we adopt the full GR framework in ZLY15, which can simulate both the motion of the particle around the massive black hole and the propagation of the photons emitted from the particle ( star or pulsar) to the observer in pure Kerr metric. The framework is briefly described below, for the details we refer the reader to ZLY15. We expand the framework to include the TOAs of pulsar in the simulation, as well as the motion of the whole system with respect to the solar system. Details of these are given in Section 2.2 and Section 2.3 , respectively.

Alternative approaches would be a perturbative treatment similar to a post-Newtonian expansion (cf., Wex 1995) or a mixed perturbative and numerical approach (Angélil et al. 2010). The present method, though complex in implementation, is conceptually simpler than these approaches.

\subsection{The full Kerr metric framework}

We assume a Kerr spacetime around the GC, with a central mass of $M_{\bullet}=4 \times 10^{6} M_{\odot}$ corresponding to a gravitational radius of $r_{\mathrm{g}} \simeq 0.04 \mathrm{AU} \simeq 5 \mu \mathrm{as} \simeq 2 \times 10^{-4} \mathrm{mpc}$. The GC distance we take to be $R_{\mathrm{GC}}=8 \mathrm{kpc}$.

Orbits of pulsars are integrated in Boyer-Lindquist coordinates under a full Kerr metric. The equations of motion are given in Equations 19-22 in ZLY15. The orbital elements of the pulsar, being the semimajor axis $a_{\star}$, eccentricity $e_{\star}$, inclination $I_{\star}$, the position angle of the ascending node $\Omega_{\star}$, angle of periapsis $\omega_{\star}$, and the true anomaly $f_{\star}$ (or equivalently the time of the pericenter passage $t_{0 \star}$ ), are all defined with respect to the sky plane. The spin direction of the MBH is defined by two angles: $i$ and $\epsilon$. Here $i$ is the inclination to the line of sight, while $\epsilon$ is the position angle with respect to a reference direction on the sky. All these angles are illustrated in Figure 1

We adopt the backward light-tracing technique described in ZLY15 to calculate the observed position $\mathbf{r}_{\star}$ of the target pulsar in the sky plane (with respect to the position of the MBH). Here $\mathbf{r}_{\star}=($ R.A., decl.), where $\mathrm{R}$.A. is the right ascension and decl. is the declination. In the simulations, R.A. $=-\alpha r_{\mathrm{g}} / R_{\mathrm{GC}}$, decl. $=\beta r_{\mathrm{g}} / R_{\mathrm{GC}}$, where $\alpha$ and $\beta$ are dimensionless impact parameters of the pulse from the pulsar projected into the sky plane. These two values are given by

$$
\begin{aligned}
\lambda & =-\alpha \sin i, \\
q^{2} & =\beta^{2}+\left(\alpha^{2}-a^{2}\right) \cos ^{2} i .
\end{aligned}
$$

Here $\lambda=L_{z} / E_{m}$ and $q^{2}=Q / E_{m}^{2}$ are two constants of motion for the pulse trajectory, $L_{z}$ is the azimuthal angular momentum, $Q$ is the Carter constant and $E_{m}$ is the energy of the photon at infinity.

Similar to the case of stars, we can also obtain the corresponding relativistic redshift $Z_{\star}$ of the pulse as

$$
1+Z_{\star}=\frac{p_{\mu} U^{\mu}}{p_{\nu}^{\text {obs }} U_{\text {obs }}^{\nu}}
$$

where $p^{\mu}$ is the four momentum of the pulse photon and 

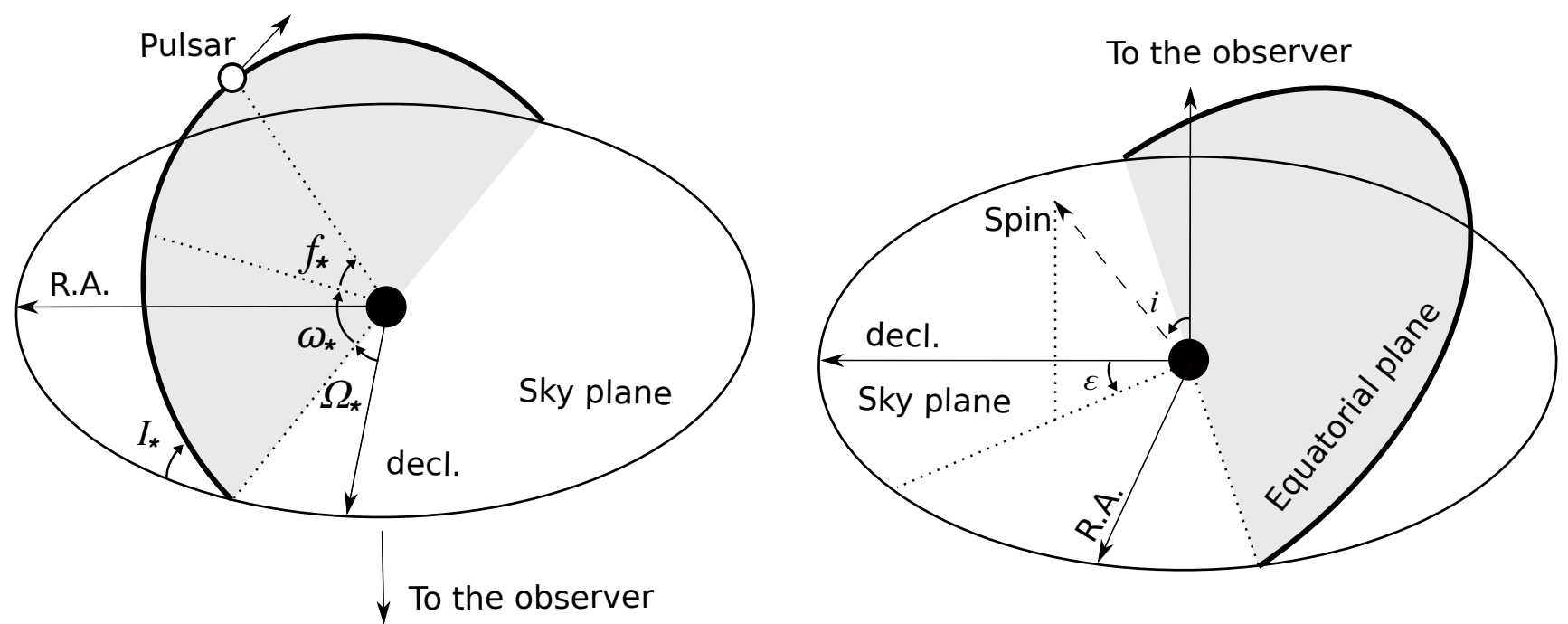

Figure 1. Illustration of the angles used in this work. Left panel: Angles of pulsar's orbit defined in the sky plane (see also Eisenhauer et al. 2005). Here $I_{\star}, \Omega_{\star}, \omega_{\star}$ and $f_{\star}$ are the orbital inclination, position angle of the ascending node, angle of the periapasis, and true anomaly, respectively. The orbital elements of the pulsar are defined with respect to the position of the MBH, and here the MBH is located at R.A. $=0$ and decl. $=0$. Right panel: Angles defined for the spin orientation: $i$ is the spin inclination with respect to the line of sight, while $\epsilon$ is the position angle of spin axis with reference to the direction of decl. To make the angles easier to see, the two panels use different orientations - note the direction to the observer in each case.

$U^{\mu}$ is the four velocity of the pulsar. The redshift is not directly measurable for pulsars, since the intrinsic frequency is not known, but can be measured as it essentially is the derivative of the TOA (see details in Section 3.4.3).

As we adopt the full Kerr metric, all the various GR effects on the orbital motion of the target pulsar around the Kerr $\mathrm{MBH}$ and on the propagation of the photons from the pulsar to the observer, are simultaneously included in the mock observables of the target pulsar, e.g., the TOA and the apparent motion in the sky plane.

\subsection{The motion of $\operatorname{Sgr} A^{*}$}

The position and the proper motion of the GC $\mathrm{MBH}$ in the sky plane is usually indicated by its radio counterpart, i.e., Sgr A*. The previous framework of ZLY15 assumes that the GC MBH remains fixed in the sky. Here we include the proper motion of the $\mathrm{MBH}$ in calculating the apparent motion of the pulsars. The apparent proper motion of a pulsar in the sky plane is the sum of the motion of the $\mathrm{MBH}$ and also its relative motion with respect to the MBH. By including the motion of the $\mathrm{MBH}$ in the modeling, the apparent motion of the pulsar can then be used directly in constraining the $\mathrm{MBH}$ parameters. The removal of the motion of Sgr A* itself by some independent measurements is no longer needed. The relative acceleration between Sgr A* and the Sun is neglected.

By the method described in Section 2.1, we can obtain the evolution of the relative sky position $\mathbf{r}_{\star}$ between the pulsar and the MBH. Suppose that Sgr A* has a constant velocity in the sky plane $\mathbf{V}_{\bullet}=\left(V_{x}, V_{y}\right)$. The apparent sky position of the $\operatorname{Sgr} A^{*}, \mathbf{R}_{\bullet}$, is then given by

$$
\mathbf{R}_{\star}=\mathbf{r}_{\star}+\mathbf{R}_{\bullet}, 0+\mathbf{V}_{\bullet} t_{\star} .
$$

Here $\mathbf{R}_{\bullet}, 0=\left(X_{0}, Y_{0}\right)$ is the initial position of the Sgr $\mathrm{A}^{*}$ at the beginning of the observation, $t_{\star}$ is the coordinate time of the pulsar. Note that the line of sight velocity $\left(V_{z}\right)$ of the MBH respective to the Sun or the Local Standard of Rest (LSR) can not be constrained in our model as it is absorbed in the apparent pulse frequency. Here we simply assume that $V_{z}=0$.

The proper motion of Sgr A* has been measured by a number of observations (e.g., Reid \& Brunthaler 2004; Ghez et al. 2008). The former give values of $18 \pm 7 \mathrm{~km} \mathrm{~s}^{-1}$ in the galactic longitude and $-0.4 \pm 0.9 \mathrm{~km} \mathrm{~s}^{-1}$ in the galactic latitude (assuming $R_{0}=8 \mathrm{kpc}$ ). If the motions of the solar system and the LSR are included, the apparent motion of the Sgr A* is $-241 \pm 15 \mathrm{~km} \mathrm{~s}^{-1}$ and $-7.6 \pm 0.7 \mathrm{~km} \mathrm{~s}^{-1}$ in the galactic longitude and latitude, respectively. In our MCMC model-fitting procedure, in principle the position and the velocities of $\mathrm{Sgr} \mathrm{A}^{*}$ can be arbitrarily selected. However, to mimic the picture expected in future observations, we adopt the values $V_{x}=-3.151$ mas yr $^{-1}, V_{y}=-5.547$ mas yr $^{-1}$, both with respect to the Sun. At the beginning of each simulation we set $X_{0}=0$ mas and $Y_{0}=0$ mas, but this makes no difference to the results. For simplicity, we ignore the apparent evolution of the geometric orientation of the orbital plane due to the proper motion of the barycenter of the pulsar-MBH binary (See Kopeikin 1996).

\subsection{The timing of pulsars}

In the local frame of the pulsar, the proper time of the pulsar is given by $\tau=\xi s$, where $s$ is the affine parameter (e.g., Equations 19-22 of ZLY15) and $\xi=m / E_{m}, m$ and $E_{m}$ being the rest mass and the energy of the pulsar respectively. In the local frame of pulsar, suppose that the pulse frequency is $\nu_{0}$ at proper time $\tau_{0}$ and the first derivative of the pulse with respect to proper time is given by $\dot{\nu}_{0}$, then in the first order approximation, the phase of the emission $\phi$ for a given proper time $\tau$ is

$$
\phi(\tau)=\nu_{0}\left(\tau-\tau_{0}\right)+\frac{\dot{\nu}_{0}}{2}\left(\tau-\tau_{0}\right)^{2} .
$$

If we write $\nu=\nu_{0}+\dot{\nu}_{0}\left(\tau-\tau_{0}\right) / 2$ then we can also express the above equation as $\phi(\tau)=\nu\left(\tau-\tau_{0}\right)$. The zero of proper time $\tau_{0}$ does not affect the results, so for simplicity we set $\tau_{0}=0$. Note that when $\phi$ is exactly an 
integer, the corresponding value of $\tau$ is the proper time of emission in the local frame of pulsar. Unless otherwise specified, we assume that $\nu_{0}=2 \mathrm{~Hz}$ (spinning frequency $0.5 \mathrm{~s})$ and $\dot{\nu}_{0}=-10^{-15} \mathrm{~s}^{-2}(\sim 31.6 \mathrm{nHz}$ per year $)$, which are typical values for a normal pulsar. The detection of millisecond pulsars is unlikely (e.g., Cordes \& Lazio 1997; Macquart et al. 2010).

When a pulse is emitted at the proper time $\tau$, whenever $\phi$ in Equation (4) is an integer, we can obtain the corresponding coordinate time $t_{\star}$ of the pulsar from the equations of motion (Equations 19-22 in ZLY15). According to the light-tracing method described in ZLY15, we can trace the photon from a distant observer (located at distance $r_{0}=10^{8} r_{\mathrm{g}}$ ) to the position of the pulsar, and the time of propagation $t_{\text {prop }}$ can be obtained. For simplicity we shift the time of propagation, i.e., $t_{\mathrm{prop}} \rightarrow t_{\mathrm{prop}}-r_{0} / c$, such that $t_{\text {prop }}$ is approximately the additional time of propagation used for the pulse crossing the pulsar-MBH binary respect to the position of the $\mathrm{MBH}$. The observed TOA $t_{\text {arr }}$ of the pulse is then given by

$$
t_{\text {arr }}=t_{\star}+t_{\text {prop }} \text {. }
$$

We do not include the additional time corrections due to the scatterings of the interstellar medium and the translations from the barycenter of Solar system to the local time of observational stations on earth. For more details of these corrections see Edwards et al. (2006).

\section{THE RELATIVISTIC MOTION OF HYPOTHETICAL PULSARS IN THE GALACTIC CENTER}

With the numerical methods described in Section 2 , here we investigate the GR signals, especially the spininduced effects in the observables of hypothetical pulsars around the GC MBH. Let $\delta_{a} Y$ be the spin-induced difference on $Y$, here $Y$ is any quantity of interest, e.g., $t_{\mathrm{arr}}$, $\mathbf{R}_{\star}$, and etc. We can estimate $\delta_{a} Y$ by performing simulations with and without the spinning of the $\mathrm{MBH}$ and then estimate the difference in $Y$. More explicitly, $\delta_{a} Y$ is defined by

$$
\delta_{a} Y=Y(a, \boldsymbol{\vartheta})-Y(0, \boldsymbol{\vartheta}) \simeq \frac{\partial Y}{\partial a} a
$$

Here $\boldsymbol{\vartheta}$ stands for the initial values of all the parameters in the simulation except the MBH spin $a$. For a given observational duration $T_{\text {tot }}$, we define the spin-induced effects per orbit as $\overline{\delta_{a} Y}$, which is given by

$$
\overline{\delta_{a} Y}=\frac{P}{T_{\mathrm{tot}}}\left[\int_{0}^{T_{\mathrm{tot}}} \frac{1}{T_{\mathrm{tot}}}\left|\delta_{a} Y\right|^{2} d t_{\mathrm{arr}}\right]^{1 / 2},
$$

Here $P$ is the orbital period. We estimate $\overline{\delta_{a} Y}$ by performing simulations with duration of $T_{\text {tot }}=3 P$. As roughly $\delta_{a} Y \propto t_{\text {arr }} / P$, the values defined above are approximately independent on the observational duration.

If not otherwise specified, we always adopt $i=45^{\circ}$ and $\epsilon=180^{\circ}$ for the $\mathrm{MBH}$ spin orientation.

In the following section, we discuss plausible orbits of the pulsars in GC and assume the existence of some hypothetical pulsars within $\lesssim 1000$ AU. The mock apparent position, TOA and the corresponding spin-induced effects for these example pulsars are described in Sections $3.2,3.3$ and 3.4 respectively.
Table 1

Orbital parameters for example pulsars

\begin{tabular}{lccccccc}
\hline \multirow{2}{*}{ Name } & \multicolumn{2}{c}{$a_{\star}$} & \multirow{2}{*}{$e_{\star}$} & $I_{\star}$ & $\Omega_{\star}$ & $\omega_{\star}$ & $f_{\star}$ \\
\cline { 2 - 4 } & $\mathrm{AU}^{a}$ & $r_{\mathrm{g}}{ }^{b}$ & & & & & \\
\hline S2-like & 984 & 24949 & 0.88 & $135^{\circ}$ & $225^{\circ}$ & $63^{\circ}$ & $180^{\circ}$ \\
S0-102-like & 848 & 21500 & 0.68 & $151^{\circ}$ & $175^{\circ}$ & $185^{\circ}$ & $180^{\circ}$ \\
Ea & 300 & 7606 & 0.88 & $135^{\circ}$ & $225^{\circ}$ & $63^{\circ}$ & $180^{\circ}$ \\
Eb & 300 & 7606 & 0.88 & $151^{\circ}$ & $175^{\circ}$ & $185^{\circ}$ & $180^{\circ}$ \\
\hline
\end{tabular}

Note. - $a$ in unit of AU.

$b$ in unit of the gravitational radius $r_{\mathrm{g}}=G M_{\bullet} / c^{2}$ and the $\mathrm{MBH}$ mass $M_{\bullet}$ is assumed to be $4 \times 10^{6} M_{\odot}$.

\subsection{The orbits of hypothetical pulsars}

To explore the spinning the GC MBH the orbiting pulsar should be close enough, e.g., at comparable distance to or closer than the currently detected star $\mathrm{S} 2 / \mathrm{S} 0-2$ or S0-102 $\left(a_{\star} \lesssim 10^{3} \mathrm{AU}\right)$. These pulsars and their progenitors are unlikely to have formed in situ, as the tidal forces of the $\mathrm{MBH}$ is quite strong. Nor is it likely that they migrated to these distance through secular dynamical process (e.g., by two body relaxation processes) as the corresponding timescale is much longer than the lifetime of the pulsar and the progenitor stars. A plausible model is that their progenitors are captured by the MBH through tidal break up of binary stars (Zhang et al. 2014). By including the supernova kick, secular dynamic relaxations and gravitational wave decay, Zhang et al. (2014) estimated that a number of $\sim 10-15$ pulsars are expected to be hidden within distance of $1000 \mathrm{AU}$ from the black hole.

Zhang et al. (2014) show that the orbits of the hypothetical innermost pulsars are expected to have $a_{\star}=$ $100-500 \mathrm{AU}$ and have distribution of eccentricity similar to those of the currently detected S-stars. Currently, the two closest S-stars from the GC MBH are the S2/S02 (Gillessen et al. 2009; Ghez et al. 2008; Gillessen et al. 2017) and the S0-102 (Mever et al. 2012). It is possible in some scenarios that the innermost pulsars have distances similar to these stars (Zhang et al.|2014), thus, it would be also quite interesting to see the spin-induced effects in timing of these pulsars, which are relatively away from the $\mathrm{MBH}$ and in periods of $11 \sim 15 \mathrm{yr}$.

Based on these results, we select four example pulsars, of which the orbital parameters are shown in Table 1 . We notice that the GR and spin-induced effects depend on the orbital orientations, and thus two different orbital orientations of the example pulsars are assumed. We assume S2-like and S0-102-like pulsar, with the orbits similar to the currently detected S2/S0-2 and S0-102, respectively. Two miniature versions of these two pulsars, the pulsar "Ea" and "Eb" are with orbits similar to S2/S0-2 and S0-102, respectively, but both with $a_{\star}=300 \mathrm{AU}$ (period of $\sim 2.6 \mathrm{yr}$ ) and $e_{\star}=0.88$. Note that the orbital semimajor axis or the eccentricity of these example pulsars could vary according to the problems discussed in this work. Pulsars with orbits of $a_{\star}<100 \mathrm{AU}$ and $e_{\star}>0.98$ may decay their orbits by gravitational wave within 10Myrs (according to Peters (1964)). We avoid these pulsars as we have not included the effects of gravitational wave decay in the simulation.

\subsection{The apparent motion and orbital precession of} pulsar 

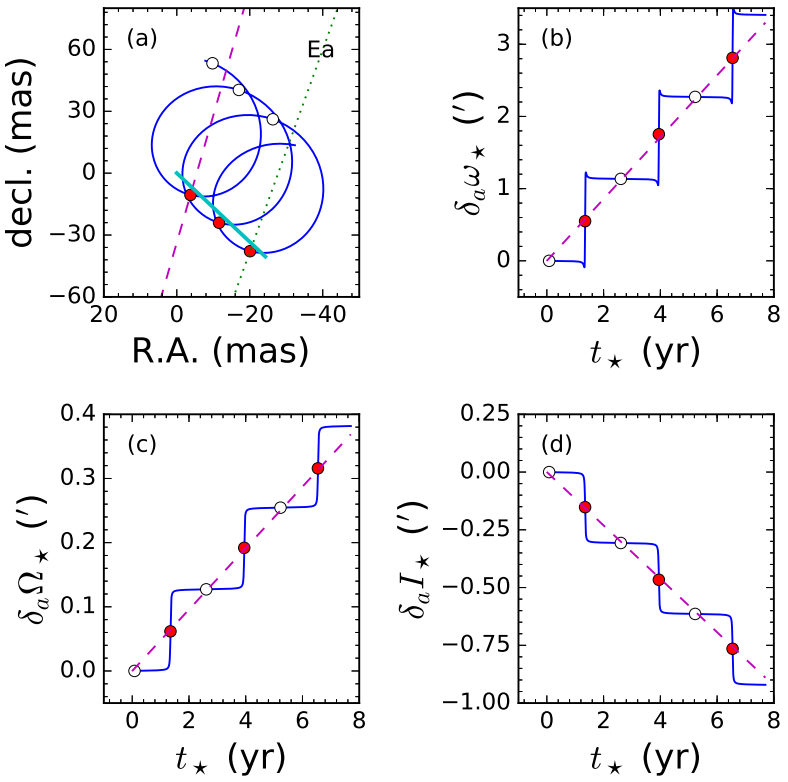

Figure 2. Panel (a): Apparent motion of the example pulsar Ea in the the sky plane over three orbits $(\sim 8 \mathrm{yr})$. The magenta dashed and green dotted lines are the direction of the instantaneous eccentricity vector of the pulsar at the beginning and the end of the simulation, respectively. The cyan line shows the trajectory of the $\mathrm{MBH}$ in the GC. Panels $(\mathrm{b}-\mathrm{d})$ The spin-induced orbital precession (in arcmin) in the angle of periapsis $\omega_{\star}$, the position angle of ascending node $\Omega_{\star}$ and the inclination $I_{\star}$. The dashed magenta line show the theoretical expectations from Equation (12). The red solid and white empty circle in all panels show the position of the pericenter and apocenter passage points.

The top left panel of Figure 2 show the apparent motion in the sky plane of the example pulsar Ea in three orbits. The proper motion of the $\mathrm{Sgr} \mathrm{A}^{*}$ is quite significant. As the pulsar is moving along with the Sgr A*, its apparent trajectory in the sky plane shows a spiral-like pattern. For the pulsar Ea, the transient velocity can be up to $\sim 100 \mathrm{mas} / \mathrm{yr}$ near the pericenter and $\sim 10 \mathrm{mas} / \mathrm{yr}$ near the apocenter, this suggests that the proper motion of a pulsar can be easily measured by future long-baseline radio telescopes.

Both the TOA and the apparent positions of pulsars contain relativity-induced effects. The Schwarzschild effects introduce precession only of the pericenter. Over one orbit, the precession is given by (Wex \& Kopeikin 1999)

$$
\delta_{S} \omega=6 \pi G M_{\bullet} c^{-2} a_{\star}^{-1}\left(1-e_{\star}^{2}\right)^{-1}
$$

The precession of orbits in a Kerr metric is well known. If $\omega_{\bullet}, \Omega_{\bullet}, I_{\bullet}$ are orbital elements with respect to the equatorial plane of a spinning black hole, then over one orbit, the first two angles precess by (e.g., Lense \& Thirring 1918; Wex \& Kopeikin 1999)

$$
\begin{aligned}
& \delta_{a} \Omega_{\bullet}=4 \pi a\left(G M_{\bullet}\right)^{3 / 2} c^{-3} a_{\star}^{-3 / 2}\left(1-e_{\star}^{2}\right)^{-3 / 2} \\
& \delta_{a} \omega_{\bullet}=-3 \delta_{a} \Omega_{\bullet} \cos I_{\bullet}
\end{aligned}
$$

to leading order. In observers' coordinates these preces- sions transform (cf. Zhang et al. 2015; Yu et al. 2016)

$$
\begin{aligned}
\delta_{a} I_{\star} & =\sin i \cos \left(\epsilon-\Omega_{\star}\right) \delta_{a} \Omega_{\bullet}, \\
\delta_{a} \omega_{\star} & =\frac{\sin i \sin \left(\epsilon-\Omega_{\star}\right)}{\sin I_{\star}} \delta_{a} \Omega_{\bullet}+\delta_{a} \omega_{\bullet}, \\
\delta_{a} \Omega_{\star} & =\left[\cos i-\frac{\cos I_{\star} \sin i \sin \left(\epsilon-\Omega_{\star}\right)}{\sin I_{\star}}\right] \delta_{a} \Omega_{\bullet} .
\end{aligned}
$$

For this, the direction cosine

$$
\cos I_{\bullet}=\cos I_{\star} \cos i+\sin I_{\star} \sin i \sin \left(\epsilon-\Omega_{\star}\right)
$$

must be substituted into Equations (9). Combining the Schwarszchild and the spin effects, the orbital precession can be expressed as

$$
\begin{aligned}
\omega_{\star} & =\omega_{\star 0}+\delta_{a} \omega_{\star} \frac{t_{\star}}{P}+\delta_{S} \omega \frac{t_{\star}}{P}, \\
\Omega_{\star} & =\Omega_{\star 0}+\delta_{a} \Omega_{\star} \frac{t_{\star}}{P}, \\
I_{\star} & =I_{\star 0}+\delta_{a} I_{\star} \frac{t_{\star}}{P} .
\end{aligned}
$$

Here $t_{\star} \simeq \tau \simeq t_{\mathrm{arr}}$.

In fact, analytical expectation of Equation (12) fails to trace the part of the orbital-element variations that is caused by the spin-induced effects. Panels (b-d) of Figure 2 show simulation results of spin-induced orbital precession in $\Omega_{\star}, \omega_{\star}$ and $I_{\star}$. The expectations from Equation (12) are shown in the dashed magenta lines in each panel. Due to the relatively large orbital eccentricity, the orbital precession of pulsar Ea in simulations mainly occur near the pericenter, with nearly Keplerian behavior near the apocenter. In consequence, as we will show in more detail later in Section [3.4 the spin-induced TOA difference predicted by Equation (19) below could not trace accurately those obtained by our relativistic simulations. The deviations $(\sim 10 \mathrm{~s})$ are quite apparent, considering the timing accuracies $(\lesssim 1-10 \mathrm{~ms})$ expected for future facilities, e.g., the SKA.

\subsection{The timing of the pulsars}

According to Equation (5), for a proper time $\tau$ that corresponds to the emission of a pulse, its TOA can be alternatively expressed as

$$
t_{\text {arr }}=\tau+\Delta_{\star}+\Delta_{\text {prop }}
$$

Here $\Delta_{\star}=t_{\star}-\tau$ and $\Delta_{\text {prop }}=t_{\text {prop }} . \Delta_{\star}$ translates the proper time to the coordinate time of pulsar. $\Delta_{\text {prop }}=$ $t_{\text {prop }}$ translates the time of emission of the pulse in the local coordinate to the observer's frame.

$\Delta_{\star}$ is also commonly dubbed the "Einstein delay". In the weak field approximation, $d t / d \tau \simeq 1+$ $2 G M_{\bullet} /\left(r c^{2}\right)-G M_{\bullet} /\left(2 a_{\star} c^{2}\right)$. By the method similar to Blandford \& Teukolsky (1976), the Einstein delay can be expressed as 1

$$
\Delta_{\star}=t_{\star}-\tau \simeq \tilde{\gamma}\left(\sin E^{\prime}-\sin E_{0}^{\prime}\right)+\frac{3 G M_{\bullet}}{2 a_{\star} c^{2}} t_{\star}
$$

1 Note that the expression is slightly different from Blandford \& Teukolsky (1976) and Damour \& Deruelle (1986) as we do not omit constants in the derivation. In the literature, these constants are commonly absorbed in the spin frequency of pulsars. 

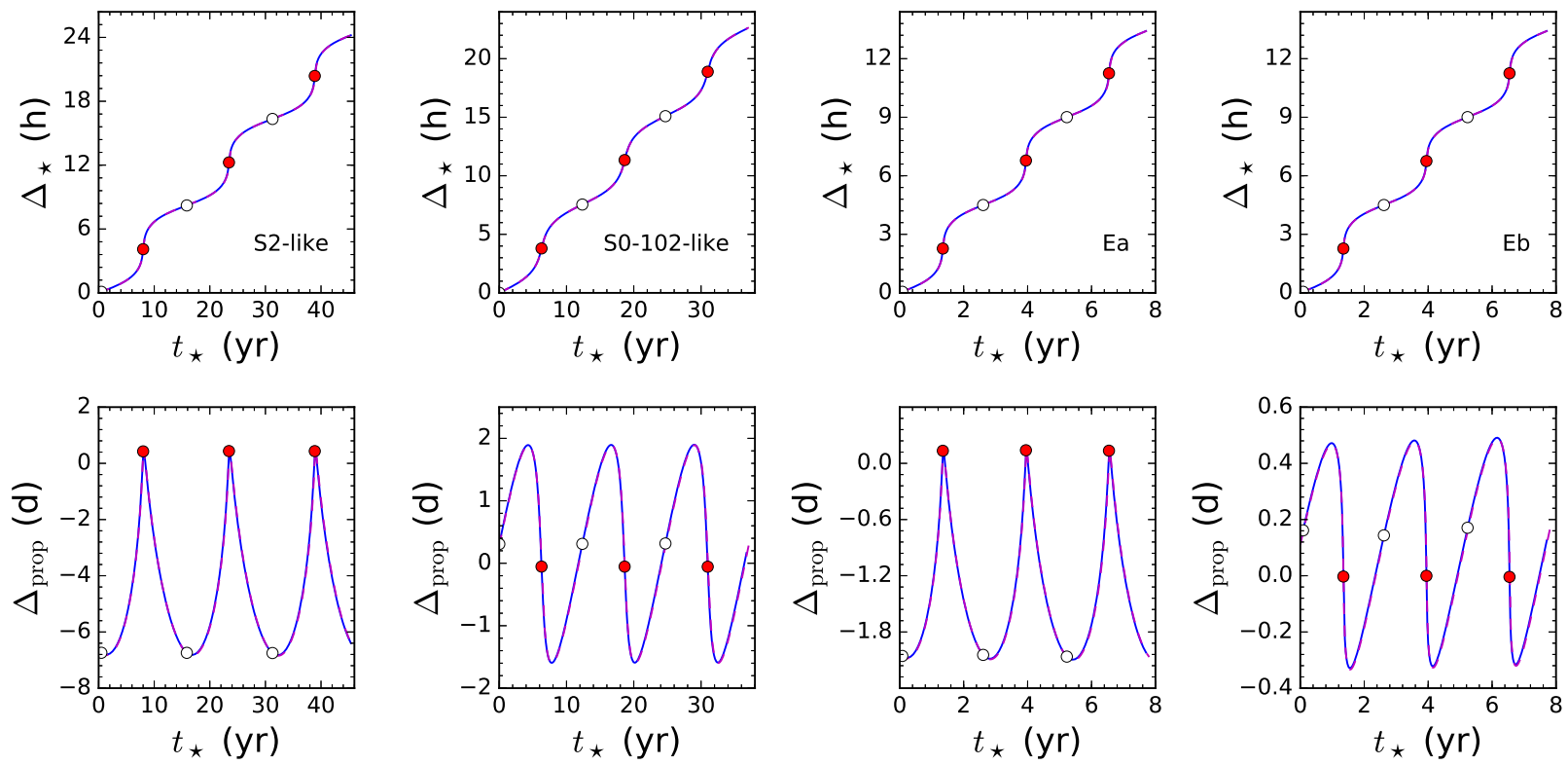

Figure 3. Top panels: The Einstein delay (in hours) which connects the proper time to the coordinate time of the pulsar; Bottom panels: The propagation time delay (in days) from a pulsar to the observer. The red solid and white empty circle show the position of the pericenter and apocenter passage points. The magenta dashed lines in the top panel shows the expectation from Equation (14), while the bottom panel from the sum of the Equation (16) and Equation (18).

Here $\tilde{\gamma}=\frac{G M_{\bullet} P e_{\star}}{\pi a_{\star} c^{2}}, P=2 \pi a_{\star}^{3 / 2}\left(G M_{\bullet}\right)^{-1 / 2}$ is the orbital period of the pulsar, $E^{\prime}$ is the eccentric anomaly which corresponds to a coordinate time $t_{\star}$, i.e.,

$$
E^{\prime}-e_{\star} \sin E^{\prime}=\frac{2 \pi}{P}\left(t_{\star}-t_{0 \star}\right)
$$

and $E_{0}^{\prime}$ is the initial value of $E^{\prime}$. From Equation (14) we can see that $\Delta_{\star}$ is contributed by an oscillation term with the magnitude given by $\tilde{\gamma}$ and a linear term which is mainly due to the relativistic time dilation.

The top panels of Figure 3 show the evolution of $\Delta_{\star}$ obtained by the simulations (blue solid lines) and Equation (14) (dashed magenta lines) for all example pulsars. We can see that the simulation results are well consistent with the analytical formula. $\Delta_{\star}$ appears to be the same for Ea and Eb as they have the same $a_{\star}$ and $e_{\star}$. In three orbits, the delay mounts up to $\sim 13.5$ hour, and oscillates in magnitude of $\tilde{\gamma} \simeq 0.84$ hour. For the S2-like (or S0-102-like) pulsar, the delay mounts up to $\sim 24$ hour ( $\sim 22$ hour $)$ in 45 years $(\sim 37$ years $)$, and oscillates in magnitude of $\tilde{\gamma} \simeq 1.4$ hour $(\tilde{\gamma} \simeq 1$ hour $)$.

In weak fields, the last term in Equation (13), $\Delta_{\text {prop}}$, is approximately the sum of the "Roemer delay" $\Delta_{R}$ and the "Shapiro delay" $\Delta_{\mathrm{S}}$, i.e., $\Delta_{\text {prop }} \simeq \Delta_{\mathrm{R}}+\Delta_{\mathrm{S}}$. The Roemer delay is the time used for a pulse propagating in a flat spacetime to the observer, which is given by (Damour \& Deruelle 1986)

$$
\Delta_{\mathrm{R}}=\tilde{\alpha}\left(\cos E^{\prime}-e_{\star}\right)+\tilde{\beta} \sin E^{\prime}
$$

Here

$$
\begin{aligned}
& \tilde{\alpha}=a_{\star} c^{-1} \sin I_{\star} \sin \omega_{\star}, \\
& \tilde{\beta}=\left(1-e_{\star}^{2}\right)^{1 / 2} a_{\star} c^{-1} \sin I_{\star} \cos \omega_{\star},
\end{aligned}
$$

The Shapiro delay is the additional time delay due to the curved spacetime, which is given by (Shapiro 1964;
Blandford \& Teukolsky 1976)

$$
\Delta_{\mathrm{S}}=\frac{2 M_{\bullet} G}{c^{3}} \ln \left[\frac{1+e_{\star} \cos f_{\star}}{1-\sin I_{\star} \sin \left(\omega_{\star}+f_{\star}\right)}\right]
$$

For the GC MBH, we have $2 M_{\bullet} G / c^{3} \sim 39.3 \mathrm{~s}$, which is also significant. Noticing that the orbit elements are precessing, thus in evaluating Equation (16) and (18) the orbital elements $\omega_{\star}$ and $I_{\star}$ are replaced according to Equation (12).

The bottom panels of Figure 3 show the evolution of $\Delta_{\text {prop }}$ of all the example pulsars. $\Delta_{\text {prop }}$ oscillates as the pulsar rotates the $\mathrm{MBH}$ periodically, and is always dominated by the Roemer delay. For example pulsar Ea and $\mathrm{Eb}$, the magnitude of the oscillation is $\sim 2.3$ day and $\sim 0.8$ day, respectively. For the S2-like pulsar and S0102-like pulsar, the oscillation is $\sim 7.4$ day and 3.4 day, respectively. The magenta lines in the left panels of Figure 3 show the model prediction from the sum of equation [16 and 18. We can see that the simulations agree with the analytical formula well 2 .

As a summary, the low order GR effects, e.g., the Einstein delay $\Delta_{\star}$ and Shapiro delay $\Delta_{\mathrm{S}}$, are both quite significant for all these example pulsars. Thus, it is expected that the low relativistic effects can be well tested if any pulsars within $\lesssim 1000$ AU from the $\mathrm{MBH}$ can be found. According to the measured Einstein and Shapiro delay, the MBH mass can also be well constrained. However,

${ }^{2}$ We find $\sim 1 \%$ difference between $\Delta_{\text {prop }}$ (Similarly for the $\Delta_{\star}$ ) obtained by our numerical method and that obtained by the analytical method. The deviation is mainly due to the difference of the metric adopted by these two methods ( the so-called "gauge effect"). As a consequence, the positions, velocities and the timing of a pulsar with the same initial conditions are slightly different. Note that the gauge effects are automatically removed by comparing the spin-induced effects derived from the numerical and the analytical methods, thus they do not affect the results shown in top right panels of Figure 4 and Figure 5 . 
Table 2

The spin-induced effects of the example pulsars per orbit

\begin{tabular}{lcccc}
\hline Name & $\overline{\delta_{a} \Delta_{\star}}$ & $\overline{\delta_{a} \Delta_{\text {prop }}}$ & $\overline{\delta_{a} t_{\text {arr }}}$ & $\overline{\delta_{a} \mathbf{R}_{\star}}$ \\
\hline S2-like & $0.067 \mathrm{~s}$ & $12 \mathrm{~s}$ & $12 \mathrm{~s}$ & $4.6 \mu$ as \\
S0-102-like & $0.024 \mathrm{~s}$ & $2.8 \mathrm{~s}$ & $2.8 \mathrm{~s}$ & $0.79 \mu \mathrm{as}$ \\
Ea & $0.22 \mathrm{~s}$ & $22 \mathrm{~s}$ & $22 \mathrm{~s}$ & $8.6 \mu \mathrm{as}$ \\
Eb & $0.15 \mathrm{~s}$ & $21 \mathrm{~s}$ & $21 \mathrm{~s}$ & $4.8 \mu \mathrm{as}$ \\
\hline
\end{tabular}
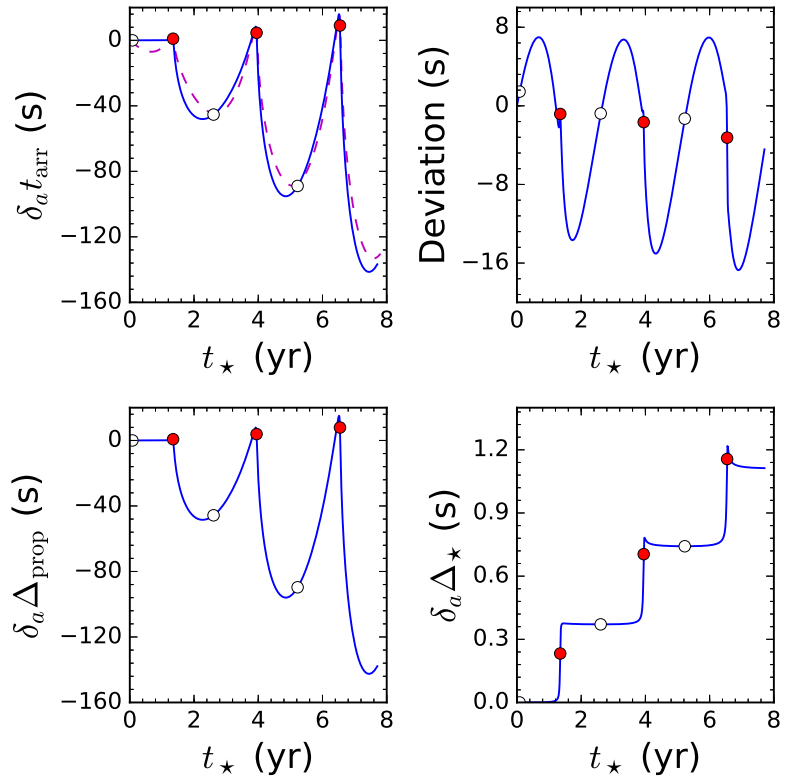

Figure 4. Spin-induced relativistic effects on the TOA $\delta_{\star} t_{\text {arr }}$ (top left panel), the propagation time delay $\delta_{a} \Delta_{\text {prop }}$ (bottom left panel) and the Einstein delay $\delta_{a} \Delta_{\star}$ (bottom right panel) of the example pulsar Ea. The blue solid line in top left panel shows the results of the numerical simulation while the dashed magenta lines show the theoretical expectations from Equation (19). Their differences as function of time are shown in top right panel. In all panels, the red solid and white empty circle show the position of the pericenter and apocenter passage points.

there is a near-degeneracy between the Einstein delay and the Roemer delay (See Equation (19)), the Einstein delay could be separated only if significant changes of the orbit orientations are present.

\subsection{The spin-induced effects}

In this section, we discuss specifically the spin-induced signals on the observables of the pulsars, i.e., the TOA and the apparent motion in the sky plane. By numerical simulations and analytical arguments, we discuss the spin-induced effects for the example pulsars which is extracted according to Equations (6) and (7). The details are shown in Section 3.4.1. We also explore the spininduced effects for pulsars in different orbital semimajor axes and eccentricities in Section 3.4.2 We notice that the spin-induced timing effects have some similarities with the corresponding redshift signals for stars. The details are shown in Section 3.4.3.

\subsubsection{Example pulsars}

The TOA $t_{\text {arr }}$ can be estimated by assuming weak fields and that the pulse propagates in a straight line connecting the observer and the pulsar. The explicit relation between $t_{\text {arr }}$ and $\tau$ in weak fields is given
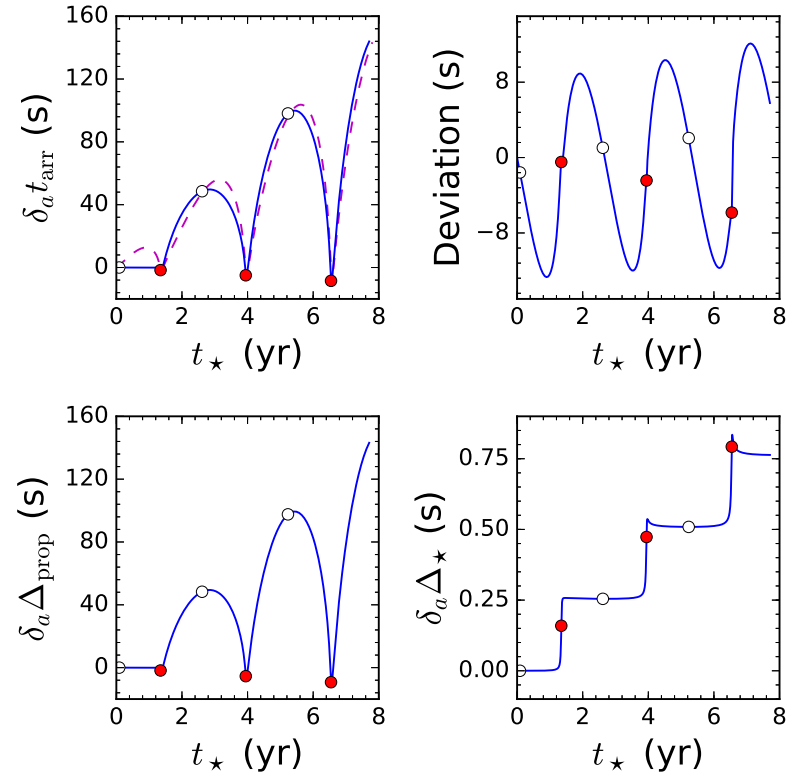

Figure 5. Similar to Figure 4 but for the example pulsar Eb.

by (Blandford \& Teukolskv 1976; Tavlor \& Weisberg 1989)

$$
\begin{aligned}
t_{\mathrm{arr}}- & \simeq\left[\tilde{\alpha}\left(\cos E-e_{\star}\right)+(\tilde{\beta}+\tilde{\gamma}) \sin E\right] \\
\times & {\left[1+\frac{2 \pi[\tilde{\alpha} \sin E-\tilde{\beta} \cos E]}{P\left(1-e_{\star} \cos E\right)}\right] }
\end{aligned}
$$

Here $E$ is the eccentric anomaly, corresponding to a given TOA $t_{\text {arr }}$ through

$$
E-e_{\star} \sin E=\frac{2 \pi}{P}\left(t_{\text {arr }}-t_{0 \star}\right)
$$

Substituting the angles in Equation (12) to Equation (19), we could get the TOA when Schwarszchild and Lense-Thirling precession are included. Note that if Equation (19) is replaced with Equation 23 from Damour \& Deruelle (1986), the spin-induced effects are quite similar, as the low order effects, e.g., the Schwarszchild-induced effects, are subtracted according to Equation (6).

According to Equation (13) we have $\delta t_{\text {arr }} \simeq \delta_{a} \Delta_{\text {prop }}+$ $\delta_{a} \Delta_{\star}$. Figure 4 shows the spin-induced difference in TOA $\delta_{a} t_{\mathrm{arr}}$, and the parts of propagation $\delta_{a} \Delta_{\text {prop }}$ and of Einstein delay $\delta_{a} \Delta_{\star}$ for the example pulsar Ea. Similar results for $\mathrm{Eb}$ are shown in Figure 5. In three orbits, the minimum and maximum TOA delay by spinning is $-141.5 \mathrm{~s}(-10.9 \mathrm{~s})$ and $15.99 \mathrm{~s}(143.9 \mathrm{~s})$ for $\mathrm{Ea}$ (for $\mathrm{Eb})$, respectively. For S2-like (S0-102-like) pulsar, they are $-79.3 \mathrm{~s}$ and $8.47 \mathrm{~s}(-5.21 \mathrm{~s}$ and $20.4 \mathrm{~s})$, respectively. Such effects are maximal around the apocenter and minimum around the pericenter, with the rapid changes mainly caused by pericenter passages. The orbital-averaged values of them are shown in Table 2. We can see that $\delta_{a} \Delta_{\star} \ll \delta_{a} \Delta_{\text {prop }}$, thus the spin-induced difference in the TOA is mainly contributed by the propagation part. Notice that $\delta_{a} \Delta_{\text {prop }}$ is approximately the sum of $\delta_{a} \Delta_{\mathrm{R}}$ and $\delta_{a} \Delta_{\mathrm{S}}$, which is the effect that the line of sight emitting position is changed by $\mathrm{MBH}$ spinning, and that the prop- 

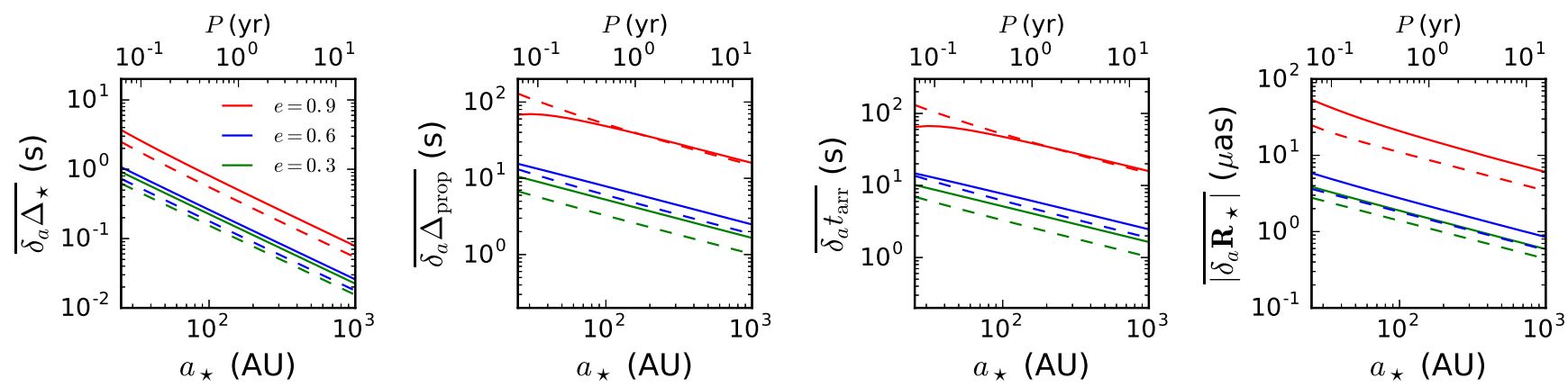

Figure 6. Spin-induced difference per orbit on the Einstein delay $\overline{\delta_{a} \Delta_{\star}}$, the propagation delay $\overline{\delta_{a} \Delta_{\text {prop }}}$, TOA $\overline{\delta_{a} t_{\text {arr }}}$ and the apparent position $\overline{\left|\delta_{a} \mathbf{R}_{\star}\right|}$ as function of the orbital semimajor axis. The solid and dashed lines are results of the example pulsar Ea and Eb, respectively, in the cases of different orbital eccentricity.

agation trajectory is changed by $\mathrm{MBH}$ spinning, respectively.

The spinning of the MBH changes the orbital motion of the pulsar compared to the case that the $\mathrm{MBH}$ is nonspinning, thus the pulsar feels a different potential and moves with different velocities, leading to difference in the Einstein delay. The Einstein delay by spin-induced effects, i.e., $\delta_{a} \Delta_{\star}$, mounts up to a maximum value of $1.2 \mathrm{~s}$ and $0.8 \mathrm{~s}$ in 8 years for $\mathrm{Ea}$ and $\mathrm{Eb}$, respectively. For S2-like and S0-102-like pulsar they are $0.37 \mathrm{~s}$ and $0.14 \mathrm{~s}$ respectively. The effects increase rapidly around the pericenter passages and remain almost constant near the apocenter. Note that both $\delta_{a} \Delta_{\text {prop }}$ and $\delta_{a} \Delta_{\star}$ of all example pulsars are quite significant compared to the typical timing accuracies of pulsar observations, e.g., $\lesssim$ $1-10 \mathrm{~ms}$. This suggests that they can be measured quite accurately by future timing observations, if any pulsars with $a_{\star} \lesssim 1000 \mathrm{AU}$ can be detected.

The model prediction of the spin-induced TOA difference can be obtained by first replacing $\omega_{\star}$ and $I_{\star}$ in Equation (12) into Equation (19), and then estimate the spin effects by Equation (6). The magenta lines in top left panel of Figure 4 and 5 show the results for Ea and $\mathrm{Eb}$, respectively. The analytical and simulation results are generally consistent, especially at the pericenter and apocenter passages. However, the discrepancies between the analytical and the simulation results are quite apparent in other regions. The maximum difference can be in orders of $\sim 10$ s (See the top right panel of Figure 4 and 5), much larger than the measurement errors expected by the future telescopes. Such discrepancies arise as in analytical formula the orbital precessions are assumed to linearly increase with time. However, they does not describe accurately the evolution compared to those obtained by our relativistic simulations (See Figure 2). These results suggest that full relativistic treatment that is presented in this work, or more sophisticated analytical models which can trace accurately the orbital precession and describing the timing signals, are necessary of the GC pulsar timing observations.

\subsubsection{Pulsars in different orbits}

In this section, we explore the dependence of the spininduced difference of observables on the distance and eccentricity of the pulsar. We perform simulations for pulsars similar to $\mathrm{Ea}$ or $\mathrm{Eb}$, but their $a_{\star}$ varies from $25-10^{3} \mathrm{AU}$ and $e_{\star}$ takes the values of $0.3,0.6$ or 0.9 .
Figure 6 shows the spin-induced effects per orbit according to Equation (7). We can see that these spin-induced timing differences show strong dependencies on the orbital distance. For example, for the pulsars similar to $\mathrm{Ea}$ (or Eb) but with $e_{\star}=0.9, \overline{\delta_{a} \Delta_{\star}}$ varies from $3.6 \mathrm{~s}$ to $0.07 \mathrm{~s}$, (or $\overline{\delta_{a} \Delta_{\star}}$ varies from $2.4 \mathrm{~s}$ to $0.05 \mathrm{~s}$ ) if $a_{\star}$ changes from $25 \mathrm{AU}$ to $10^{3} \mathrm{AU}$. Similarly, $\overline{\delta_{a} t_{\mathrm{arr}}}$ varies from $65 \mathrm{~s}$ to $16 \mathrm{~s}$ (or $\overline{\delta_{a} t_{\text {arr }}}$ varies from $130 \mathrm{~s}$ to $15 \mathrm{~s}$ ) if $a_{\star}$ changes from $25 \mathrm{AU}$ to $10^{3} \mathrm{AU}$. Approximately, $\overline{\delta_{a} \Delta_{\star}} \propto a_{\star}^{-1}$, $\overline{\delta_{a} \Delta_{\text {prop }}} \simeq \overline{\delta_{a} t_{\text {arr }}} \propto a_{\star}^{-1 / 2}$. The spin-induced timing differences depend also strongly on the orbital eccentricities. For example, $\overline{\delta_{a} t_{\text {arr }}}$ and $\overline{\delta_{a} \Delta_{\star}}$ for pulsars with eccentricities of $e_{\star}=0.9$ are about one orders of magnitude larger than those with eccentricities of $e_{\star}=0.6$ or $e_{\star}=0.3$.

The right panel of Figure 6 shows the spin-induced position difference per orbit, i.e., $\overline{\left|\delta_{a} \mathbf{R}_{\star}\right|}$, of pulsars at different orbital semimajor axes and eccentricities. For pulsar Ea with eccentricity 0.9 (or 0.3 ), $\overline{\left|\delta_{a} \mathbf{R}_{\star}\right|}$ varies from $53 \mu$ as $(3.8 \mu$ as $)$ to $17.8 \mu$ as $(0.6 \mu \mathrm{as})$ if $a_{\star}$ changes from $25 \mathrm{AU}$ to $10^{3} \mathrm{AU}$. For pulsar Eb with 0.9 (or 0.3), $\overline{\left|\delta_{a} \mathbf{R}_{\star}\right|}$ from $25 \mu$ as $(2.8 \mu \mathrm{as})$ to $3.4 \mu$ as $(0.4 \mu$ as $)$ when $a_{\star}$ varies from $25 \mathrm{AU}$ to $10^{3} \mathrm{AU}$. Taking the astrometric accuracy achievable by future telescopes as $\sim 10 \mu$ as, we can see that the signal is only measurable for pulsars with high orbital eccentricity (e.g., $e_{\star} \gtrsim 0.9$ ), or pulsars with orbital period less than one year, e.g., $a_{\star} \lesssim 100$ AU. For more details of the spin-induced position difference see Zhang et al. (2015).

Note that the orbital precession of pulsars with $a_{\star} \lesssim$ $100 \mathrm{AU}$ and $e_{\star}=0.9$ is extremely strong: The orbital precession on $\omega_{\star}$ of these pulsar can be up to $\sim 10^{\circ}$ per orbit (which is the sum of the Schwarszchild and the Lense-Thirling precession). Such strong orbital precession causes significant changes of the apparent configurations of the pulsar orbits. As consequences, $\overline{\delta_{a} t_{\text {arr }}}$ and $\overline{\left|\delta_{a} \mathbf{R}_{\star}\right|}$ for these pulsars do not strictly follow the power law relation with $a_{\star}$ (See Figure [6).

\subsubsection{The TOA and the redshift signals}

The correspondence between the timing and the redshift signal of the pulsar can be obtained as follows. For two adjacent pulses separated in the local frame of the pulsar by $\delta \tau$, the TOA is separated in the observed frame 
by $\delta t_{\text {arr }}$. When $\delta \tau \rightarrow 0$ we have

$$
\frac{d t_{\mathrm{arr}}}{d \tau}=\frac{Z_{\star}}{c}+1
$$

Therefore, the time of arrival can be estimated by integrating the above equation on the proper time $\tau$, i.e., $t_{\mathrm{arr}} \simeq \int\left(1+Z_{\star} / c\right) d \tau$. According to Equation (6), the spin-induced TOA difference is then approximately given by

$$
\delta_{a} t_{\mathrm{arr}} \simeq \frac{1}{c} \int_{0}^{T_{\mathrm{tot}}} \delta_{a} Z_{\star}(\tau) d \tau .
$$

The value of $\delta_{a} t_{\text {arr }}$ is approximately given by the integration of the spin-induced redshift difference, i.e., $\delta_{a} Z_{\star}$, over time. For pulsars comparably distant from the $\mathrm{MBH}$ as the S-stars, or closer in, considering that $\delta_{a} Z \simeq$ $1-10 \mathrm{~km} \mathrm{~s}^{-1} \simeq 10^{-5}-10^{-6} \mathrm{c}$, we have $\delta_{a} t_{\mathrm{arr}} \simeq 10-10^{2} \mathrm{~s}$ if $T_{\text {tot }}$ is of order $\sim 8 \mathrm{yr}$. These analysis results are consistent with our simulations.

\section{CONSTRAINTS ON THE SPIN PARAMETER FROM THE MOTION OF PULSARS}

In this section, we explore the achievable constraints on the spin of the GC-MBH from the TOA with and without supplementary observations of proper motions. In Section 4.1 we describe the details of the MCMC parameteric fitting method. The results for the constraints of the spin parameters and the other parameters of the $\mathrm{MBH}$, e.g., proper motion, mass and distance, are shown in Section 4.2 and 4.3 respectively. We also discuss the effects of the pulsar mass on our simulation results in Section 4.4.

\subsection{The parameter-fitting method}

We use the MCMC fitting method to study the constraints on the parameters of the pulsar-MBH binary. The initial conditions are provided by the following 17 parameters:

- Six parameters for the initial orbital elements of the pulsar: $a_{\star}, e_{\star}, I_{\star}, \Omega_{\star}, \omega_{\star}$ and $f_{\star}$.

- Two parameters describing the spin frequency of the pulsar: $\nu_{0}$ and $\dot{\nu}_{0}$.

- Four parameters for the black hole: mass $M_{\bullet}$, spin magnitude $a$, spin inclination $i$, and spin position angle $\epsilon$.

- Five parameters for the location and motion of the system with respect to the solar system: distance $R_{\mathrm{GC}}$, and proper motion $V_{\mathrm{x}}, V_{\mathrm{y}}$ and initial position $X_{0}, Y_{0}$ of the $\mathrm{MBH}$ on the sky plane. $V_{\mathrm{z}}$ does not appear as a separate parameter, as it is absorbed within $\nu_{0}$.

For a given set of mock observations, a $\chi^{2}$ value is computed and a Bayesian posterior probability function is constructed in the usual way, and then the MetropolisHasting algorithm is used to recover the parameters with uncertainties. The prior on the spin parameters are all flat distributions. The boundaries of them are $0<a<1$, $10<i<170^{\circ}, 0^{\circ}<\epsilon<360^{\circ}$. Inclinations close to $0^{\circ}$ or $180^{\circ}$ are avoided as in these cases our ray-tracing method can not calculate the trajectories of lights very accurately. For the prior on other initial conditions we assume each of them a Gaussian prior with the scatter that is about three orders of magnitude larger than its converged width obtained by the MCMC simulation. The central value of the Gaussian distribution is its input value.

Note that for all the MCMC simulations shown in Section 4.2 and Section 4.3 we set $a=0.6$ as the true value of the MBH spin. If $a=1$ is used instead, the constraints on the MBH-spin shown in Section 4.2 are about two times tighter, as the spin value is limited $0 \leq a \leq 1$.

To obtain meaningful constraints of these parameters through MCMC fitting we need to use mock samples with several times more data points than the number of free parameters. Thus, we use a total of $N=120$ mock observations (we assume one TOA and astrometric data are collected per observation) for all the MCMC simulations shown in the following sections, regardless of the observational duration assumed. For example, if the observations last for three orbits, then mock observables at 40 different epochs per orbit are collected. From Figures 4 and 5 we can see that each pericenter passage increases dramatically the magnitude of the spin-induced effects in pulsar timing. Thus, to improve the constraints on the spin of the $\mathrm{MBH}$, the time intervals between each observation is $\propto r^{-1.5}$, so that the orbit is more frequently sampled near pericenter passages.

In the case that observations consist of TOA only, $\chi^{2}=$ $\chi_{\mathrm{T}}^{2}$. Writing the $j$-th TOA as $t_{j}^{\text {arr }}, \chi_{\mathrm{T}}^{2}$ is given by (cf. Hobbs et al. 2006)

$$
\chi_{\mathrm{T}}^{2}=\sum_{j=1}^{N} \frac{\left\{\phi\left[\tau\left(t_{j}^{\mathrm{arr}}\right)\right]-\mathrm{I}_{j}\right\}^{2}}{\left(\sigma_{j}\right)^{2}} .
$$

Here

$$
\sigma_{j}=\frac{d \phi}{d t^{\mathrm{ob}}} \sigma_{T} \simeq \nu \sigma_{\mathrm{T}}
$$

is the measurement error in $\phi$, while $\sigma_{\mathrm{T}}$ is the TOA measurement error. The function $\phi\left[\tau\left(t_{j}^{\text {arr }}\right)\right]$ is provided by the full relativistic model and $\mathrm{I}_{j}$ is the integer that is closest to $\phi\left[\tau\left(t_{j}^{\text {arr }}\right)\right]$.

The expected timing accuracy of pulsar for SKA of $\sim 1$ hour integration can be down to $\sigma_{T} \simeq 100 \mu \mathrm{s}$ (Liu et al. 2012) if the frequency can be up to $\gtrsim 15 \mathrm{GHz}$, and $\sigma_{T} \simeq$ $0.1-10 \mathrm{~ms}$ if the frequency is between $\gtrsim 5 \mathrm{GHz}$ and $\lesssim 15 \mathrm{GHz}$. Considering that many factors can limit the measurement accuracy, we could expect that the TOA measurement accuracy may vary between $0.5-50 \mathrm{~ms}$. This level is assumed in our simulations. The numerical accuracy in the simulated TOA is much higher $(\lesssim 10 \mu \mathrm{s})$ so as to avoid any contamination due to numerical errors. If $\sigma_{T} \lesssim 0.5 \mathrm{~ms}$ is assumed, the constraints on the spin and other parameters of the $\mathrm{MBH}$ are correspondingly tighter.

Astrometric measurements of the pulsar, if they are available, can also be used in the MCMC runs along with the timing measurements. Suppose that each observation is denoted by $\mathbf{R}_{\star, j}^{\mathrm{ob}}$, for $j=1, \cdots, N$. The chi-square value can be expressed as $\chi^{2}=\chi_{\mathrm{T}}^{2}+\chi_{\mathrm{P}}^{2}$. Here

$$
\chi_{\mathrm{P}}^{2}=\sum_{j=1}^{N} \frac{\left(\mathbf{R}_{\star}-\mathbf{R}_{\star, j}^{\mathrm{ob}}\right)^{2}}{\sigma_{\mathrm{p}}^{2}},
$$


where $\sigma_{\mathrm{p}}$ is the astrometric error. Note that for both the mock timing and the astrometric data we assume that the measurement errors are all Gaussian.

The SKA is expected to operate with the baselines up to $3000 \mathrm{~km}$, and thus its image resolution could be up to 2 mas at $10 \mathrm{GHz}$ (Godfrey et al. 2012). The astrometric accuracy could be even higher, of order $\sim$ $10 \mu$ as (Fomalont \& Reid 2004). In this work, we simply assume that $\sigma_{\mathrm{P}}=10 \mu \mathrm{as}$. If lower astrometric accuracies are assumed, the constraints on the spin of the $\mathrm{MBH}$ are slightly affected for those pulsars with $a_{\star} \lesssim 300 \mathrm{AU}$, as the contribution of the astrometric measurements for these pulsars to the fitting are much smaller than those of the timing, i.e., $\chi_{\mathrm{T}}^{2} \gg \chi_{\mathrm{P}}^{2}$. For other parameters of the $\mathrm{MBH}$, e.g., $M_{\bullet}, R_{\mathrm{GC}}$ or the proper motions, the constraints on them will be correspondingly weaker if one sets $\sigma_{\mathrm{P}}>10 \mu \mathrm{as}$.

When only the TOAs of pulsars are used, there is no information on $\Omega_{\star}$ and $R_{\mathrm{GC}}$, hence these must be excluded from the fitting procedure. The orbital inclination $I_{\star}$ is classically degenerate with $a_{\star}$. The Einstein delay breaks this degeneracy (cf. Angélil \& Saha 2011) but as the effect is small, and a strong correlation remains, which significantly slows the convergence of the MCMC procedure. In order to concentrate on the spin, we fixed $I_{\star}$ in the simulations with TOA only, leaving 10 parameters to fit.

In the cases that both the TOA and the proper motion of pulsars are included in the MCMC fitting, we can set all the 17 parameters in the system to be free. In this case, we can constrain the proper motion of the $\mathrm{MBH}$. We find that the constraints on the spin parameters depend somewhat on whether the proper motion of the $\mathrm{MBH}$ is taken as free or not. Thus, for some of the MCMC simulations we fix the position and proper motion of the $\mathrm{MBH}$, leaving only 13 free parameters.

\subsection{Constraints on the spin parameters of the $M B H$}

By the MCMC methods described above, we perform a large number of MCMC runs to investigate the constraints on the spinning and other properties of the $\mathrm{MBH}$ by monitoring pulsars shown in Table 1 . We find that near degeneracies appear among spin parameters, however, the constraints of spin can still be very tight. Even for S2-like or S0-102-like pulsars, the spin of the $\mathrm{MBH}$ (whether the $\mathrm{MBH}$ is spinning or not) can still be probed within $\sim 4-8 \mathrm{yr}$ and $\sim 2-4 \mathrm{yr}$ in optimistic scenarios. The details of the degeneracies and the constraints on spin parameters can be found in Section 4.2.1 and 4.2.2 respectively.

We expand these studies for pulsars to other conditions of orbits and measurement accuracies. By performing a large number of MCMC simulations we discuss how the constraints on spin parameters of the $\mathrm{MBH}$ change for pulsars with different semimajor axes $a_{\star}$, eccentricities $e_{\star}$, and other parameters. For more details see Section 4.2 .3

\subsubsection{Near-degeneracies among spin parameters}

Figure 7 shows the constraints on the spin magnitude and orientation of the $\mathrm{MBH}$ from observing the hypothetical example pulsars $\mathrm{Ea}$ and $\mathrm{Eb}$ with $\sigma_{\mathrm{T}}=5 \mathrm{~ms}$.
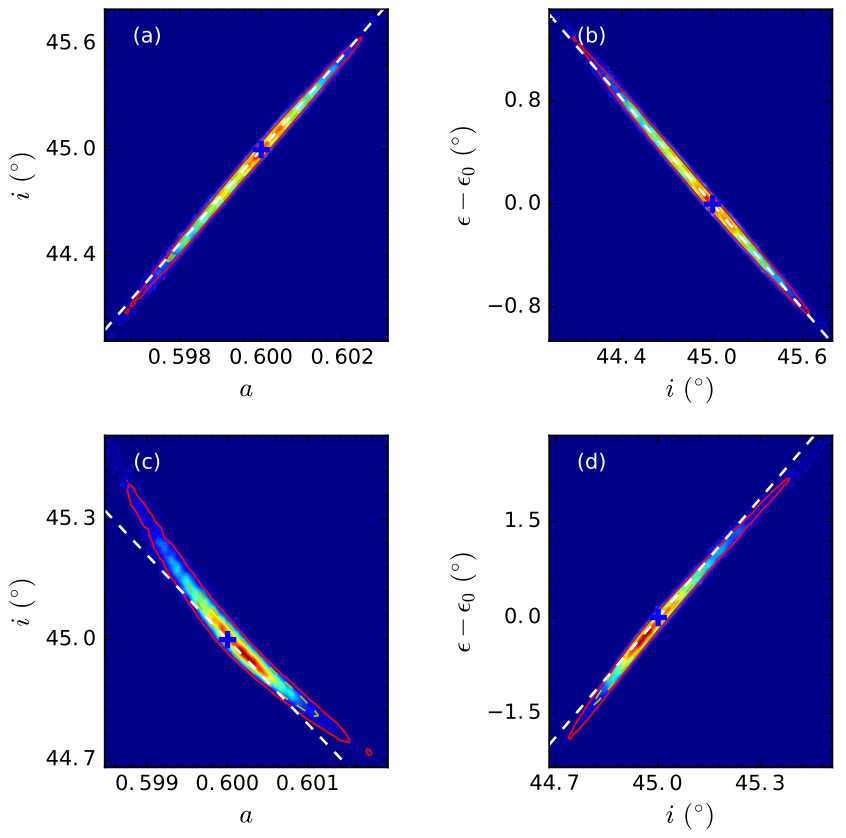

Figure 7. Constraints on the $\mathrm{MBH}$ spin parameters when only the TOAs of the pulsar are fitted. The correct values are marked by blue crosses. The assumed timing accuracy is $\sigma_{\mathrm{T}}=5 \mathrm{~ms}$. Panels (a) and (b) show results for the example pulsar Ea, while Panels (c) and (d) show results for Eb. The dashed white lines show parameter degeneracies at leading order: from Equation (29) in the left panel and from Equation (30) in the right panel. The color contour maps represent the mean likelihood of the MCMC sample, and the line contours represent the marginalized distribution. The yellow dashed and red solid lines in each panel show the $1 \sigma$ and $2 \sigma$ confidence levels respectively.

The constraints on $a, i$ and $\epsilon$ are nearly degenerate. The near degeneracies among the spin parameters when only the timing signals are used can be understood as follows. Let us write

$$
\begin{aligned}
\epsilon^{\prime} & \equiv \epsilon-\Omega_{\star}, \\
a_{x} & =a \sin i \cos \epsilon^{\prime}, \\
a_{y} & =a \sin i \sin \epsilon^{\prime}, \\
a_{z} & =a \cos i
\end{aligned}
$$

The observed TOAs of a pulsar contain information on spin-induced precessions $\Delta I_{\star}$ and $\Delta \omega_{\star}$ but not on $\Delta \Omega_{\star}$ in Equation (10). Using that fact, and substituting from Equations (9) and (11) we have

$$
\begin{aligned}
a_{x} & =\text { const } \\
a_{z}-\Gamma a_{y} & =\text { const. }
\end{aligned}
$$

Here

$$
\Gamma=\frac{1-3 \sin ^{2} I_{\star}}{3 \cos I_{\star} \sin I_{\star}} .
$$

Now using the notation $\delta Y$ for fitting uncertainty in any quantity $Y$, Equation (27) gives two relations involving $\boldsymbol{\delta} a_{x}, \boldsymbol{\delta} a_{y}$ and $\boldsymbol{\delta} a_{z}$. With the help of Equation (26) these can be rearranged as two relations

$$
\left(\Gamma \sin i-\cos i \sin \epsilon^{\prime}\right) \boldsymbol{\delta} a=-\left(\Gamma \cos i+\sin i \sin \epsilon^{\prime}\right) a \boldsymbol{\delta} i
$$

and

$$
\cos \epsilon^{\prime} \boldsymbol{\delta} i=-\left(\Gamma \sin i-\cos i \sin \epsilon^{\prime}\right) \sin i \boldsymbol{\delta} \epsilon
$$



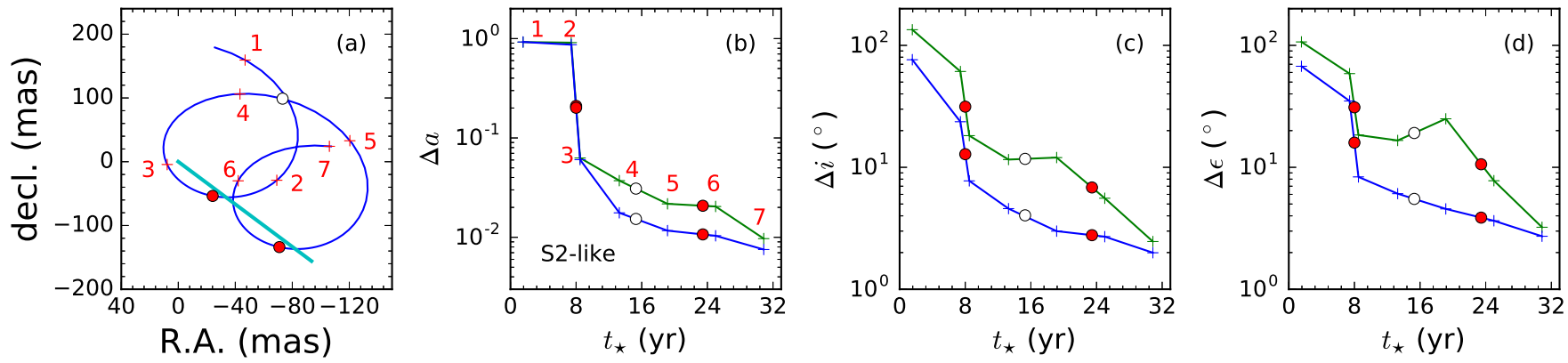

Figure 8. Constraints on the MBH spin parameters from an S2-like pulsar, as a function of the observational duration. Panel (a) show the apparent trajectories on the sky: blue curve for the pulsar and cyan line for the MBH. Initial conditions are given in Table 1 and the assumed measurement errors are $\sigma_{\mathrm{T}}=5 \mathrm{~ms}$ and $\sigma_{\mathrm{p}}=10 \mu$ as. We tried seven different observational durations, and the corresponding position of the pulsar at the end of observation are marked by numbers in Panel (a). Each of these seven cases has 120 mock observations. The accuracy on the recovered parameters are shown in Panel (b)-(d): green lines show results when only TOAs are used, while blue lines show results when both the timing and the proper motion are used. The red filled and empty white circles mark the position where the pulsar passes pericenter and apocenter respectively.
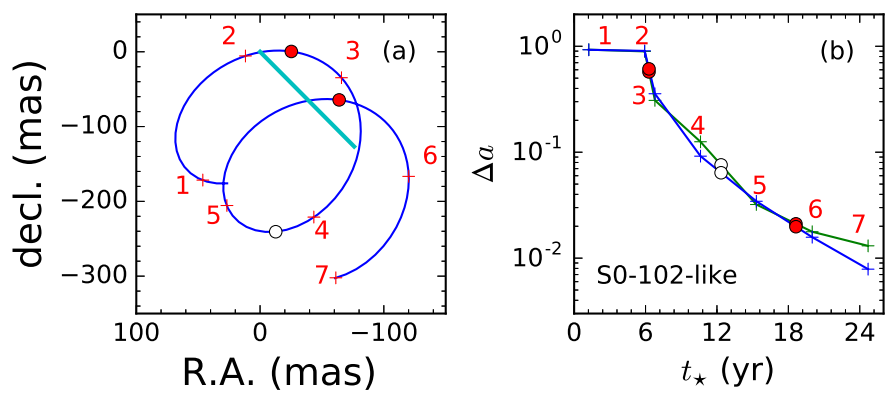

Figure 9. Similar to Figure 8 but for an S0-102-like pulsar.

Note that if $a \simeq 1$, we have the restriction that $\delta a \leq 1-a$.

The white dashed lines in Figure $\mathbf{7}$ show the predictions from Equation (29) and (30). They are in good agreement with the near-degeneracies in the simulation results. Discrepancies appear as Equations (29) and (30) are first order approximations.

From Equations (10) and (28) we can see that the degeneracies can be broken in the following four cases: (1) $\delta_{a} \Omega_{\star}$ can be inferred if proper motion of the pulsar can be measured, thus the degeneracies are broken if it is measured with considerable accuracy such that $\chi_{\mathrm{P}} \sim \chi_{\mathrm{T}}$. For an S2-like or S2-102-like pulsar the degeneracies are slightly weakened by additionally including the astrometric measurements. However, for pulsars Ea and Eb, similar degeneracies appear as the constraints are still dominated by the parts from timing, i.e., $\chi_{\mathrm{P}} \ll \chi_{\mathrm{T}}$. (2) According to Equation (28), the degeneracies are only functions of the orbital inclination $I_{\star}$ of the pulsar. Thus, the degeneracy can be broken if the apparent orbital precession of $I_{\star}$ is significant; (3) Similarly, degeneracies can be broken by combining the timing of another pulsars with different inclination. (4) The quadrupole-moment effects are strong enough that they provide another independent constraint on spin. We can see that (2) and (4) can be naturally satisfied if a pulsar has a short orbital period (e.g. $\lesssim 0.5 \mathrm{yr}$ ), or the duration of the timing observation is long enough.

\subsubsection{Results from the example pulsars}
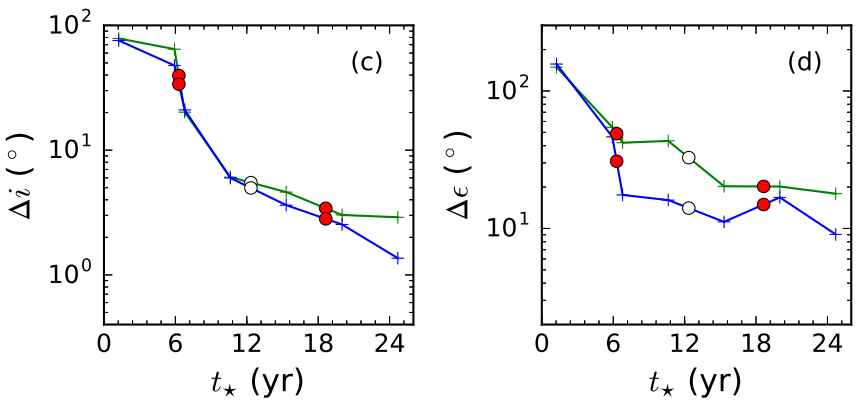

Table 3

Constraints on the spin parameters from example pulsars

\begin{tabular}{lccccccc}
\hline \multirow{2}{*}{ Name } & \multicolumn{3}{c}{ TOA $^{c}$} & & \multicolumn{3}{c}{ TOA + astrometric } \\
\cline { 2 - 4 } \cline { 6 - 8 } & $\boldsymbol{\Delta} a$ & $\boldsymbol{\Delta} i$ & $\boldsymbol{\Delta} \epsilon$ & & $\boldsymbol{\Delta} a$ & $\boldsymbol{\Delta} i$ & $\boldsymbol{\Delta} \epsilon$ \\
\hline S2-like $^{a}$ & 0.0098 & $2.5^{\circ}$ & $3.2^{\circ}$ & & 0.0075 & $2.0^{\circ}$ & $2.7^{\circ}$ \\
S0-102-like $^{a}$ & 0.0130 & $2.9^{\circ}$ & $17.9^{\circ}$ & & 0.0079 & $1.4^{\circ}$ & $9.1^{\circ}$ \\
$\mathrm{Ea}^{b}$ & 0.0046 & $1.2^{\circ}$ & $1.7^{\circ}$ & & 0.0044 & $1.2^{\circ}$ & $1.7^{\circ}$ \\
$\mathrm{Eb}^{b}$ & 0.0023 & $0.5^{\circ}$ & $3.3^{\circ}$ & & 0.0033 & $0.7^{\circ}$ & $4.7^{\circ}$ \\
\hline
\end{tabular}

Note. - ${ }^{a}$ Constraints from an S2-like or S0-102-like pulsar in two orbits, corresponding to $\sim 30 \mathrm{yr}$ or $\sim 24 \mathrm{yr}$, respectively.

${ }^{b}$ Constraints from $\mathrm{Ea}$ or $\mathrm{Eb}$ in three orbits, corresponding to $\sim 8 \mathrm{yr}$.

${ }^{c}$ Using only the timing of pulsars.

${ }^{d}$ Using both the timing and the apparent motion of pulsars.

Although near degeneracies appear among spin parameters, pulsars can still deliver very tight constraints on the spin parameters, even though the timing accuracy, i.e., $\sigma_{\mathrm{T}}=5 \mathrm{~ms}$, is not the most optimistic value. To quantify the constraints on any quantity of interest $Y$, let $Y_{2+}$ and $Y_{2-}$ be the upper and lower $2 \sigma$ confidence limits. Then let

$$
\Delta Y=Y_{2+}-Y_{2-}
$$

will be the $2 \sigma$ range of $Y$. The constraints $\Delta Y$ on the spin of the $\mathrm{MBH}$ from all the example pulsars are given in Table 3 . We can see that $\boldsymbol{\Delta} a, \boldsymbol{\Delta} i$ and $\boldsymbol{\Delta} \epsilon$ are of order $10^{-3}-10^{-2}, 0.5-3^{\circ}, 1-20^{\circ}$, respectively.

For an S2-like or S0-102-like pulsar, we find that the constraints of spin from observations over two orbital 
periods, i.e., $\sim 30 \mathrm{yr}$ or $\sim 25 \mathrm{yr}$, are also tight. Figure 8 and Figure 9 show the constraints on the spin parameters as a function of the observational duration for S2-like and S0-102-like pulsars, respectively. For such pulsars we can see that the spin of the MBH can be constrained by $\boldsymbol{\Delta} a \sim 0.1, \boldsymbol{\Delta} i \sim 20-30^{\circ}, \boldsymbol{\Delta} \epsilon \sim 20-40^{\circ}$ within $\sim 4-8 \mathrm{yr}$. The constraints on the spin can be correspondingly tighter if the observations last longer than $\sim 8-10 \mathrm{yr}$. If proper motions with accuracies around $\sigma_{\mathrm{p}}=10 \mu$ as can also be collected, the constraints could be tighter, by weakening the degeneracies among the spin parameters due to the timing measurement (See Section 4.2.1). The constraints become $\Delta a \sim 0.1$, $\Delta i \sim 5-20^{\circ}, \Delta \epsilon \sim 10-30^{\circ}$ within $\sim 8$ yr.

It is apparent that the most significant improvements on the constraints occur after pericenter passages. In the most optimistic case, the observation starts near the pericenter passages of the pulsar, in which case the spin can be constrained within $\sim 2-4$ yr for both the S2-like and S0-102-like pulsars. All this is for a relatively low timing accuracy, i.e., $\sigma_{T}=5 \mathrm{~ms}$. In reality the timing accuracy could be accumulated to be $\sigma_{T} \lesssim 0.1-1 \mathrm{~ms}$. Thus the constraint of spin can be as fast as about $2-3 \mathrm{yr}$ in the most optimistic cases.

The constraints on spin parameters (especially the orientations) seem to become slightly weaker when the observation is before the second pericenter passage (see Figure 8 and Figure 91). The reason is that we have fixed the number of observational samples at 120, instead of accumulating with time. The spin-induced signals of the pulsar between the second apocenter and the next pericenter decrease as a function of time (see top left panel of Figure 4). This makes the overall spin-induced signals smaller if the number of data points is fixed.

\subsubsection{General cases}

If $\chi=\chi_{2}^{Y}$ denotes the value where $Y$ is at its $2 \sigma$ boundary value obtained from the MCMC runs, according to Equation (23) and (25), we have, approximately,

$$
\begin{aligned}
& \left(\chi_{2}^{Y}\right)^{2} \simeq \frac{N}{T_{\text {tot }}} \int_{0}^{T_{\text {tot }}}\left\{\frac{\left[\phi\left(Y_{0}+\Delta Y / 2, t_{\text {arr }}\right)-\phi\left(Y_{0}, t_{\text {arr }}\right)\right]^{2}}{\nu_{0}^{2} \sigma_{\mathrm{T}}^{2}}\right. \\
+ & \left.\frac{\left[\mathbf{R}\left(Y_{0}+\Delta Y / 2, t_{\text {arr }}\right)-\mathbf{R}\left(Y_{0}, t_{\text {arr }}\right)\right]^{2}}{\sigma_{\mathrm{p}}^{2}}\right\} d t_{\text {arr }} \\
\simeq & \frac{\Delta Y^{2}}{4}\left[\frac{1}{\nu_{0}^{2} \sigma_{\mathrm{T}}^{2}}\left(\frac{d \phi}{d Y}\right)^{2}+\frac{1}{\sigma_{\mathrm{p}}^{2}}\left(\overline{\frac{d \mathbf{R}}{d Y}}\right)^{2}\right] N\left(\frac{T_{\text {tot }}}{P}\right)^{2}
\end{aligned}
$$

Here $N$ is the total number of epochs with data, $P$ is the orbital period and $T_{\text {tot }}$ is the duration of the observation. $\frac{\overline{d \phi}}{d Y}$ and $\overline{\frac{d \mathbf{R}}{d Y}}$ are the averaged derivatives per orbit defined similar to Equation 7.

Thus, if only the TOAs are used, we have

$$
\Delta Y \propto\left(\frac{d \phi}{d Y}\right)^{-1} \nu_{0} \sigma_{\mathrm{T}} N^{-1 / 2}\left(\frac{T_{\text {tot }}}{P}\right)^{-1}
$$

We can see that the constraint on $Y$ is approximately proportional to $N^{-1 / 2}$ and $\left(T_{\text {tot }} / P\right)^{-1}$, i.e., the constraints will be improved if more epochs are observed and if the observations last for a longer time. Also, the constraints can be improved if the intrinsic spinning frequency of the pulsar $\nu_{0}$ are higher. Note that $N$ should be much larger than the number of free parameters in MCMC simulations to ensure a meaningful fit.

We also explore the constraints on the spin parameters for pulsars with different $a_{\star}, e_{\star}$. Figure [10] shows the constraints on spin from pulsars similar to Ea, but with various $a_{\star}$, assuming $\sigma_{\mathrm{T}}=5 \mathrm{~ms}$. We can see that $\Delta a=0.002-0.006, \Delta i=0.6-2.0^{\circ}, \Delta \epsilon=0.9-3.0^{\circ}$ (or $\left.\Delta a=0.002-0.005, \Delta i=0.6-1.0^{\circ}, \Delta \epsilon=0.9-2.0^{\circ}\right)$ if only the TOAs are used (or if both the TOAs and the apparent positions are used), when $a_{\star}$ varies from $50 \mathrm{AU}$ to $600 \mathrm{AU}$. We find that $\boldsymbol{\Delta} a$ is approximately proportional to $a_{\star}^{1 / 2}$. It can be understood, as according to Equation (33), approximately $\Delta a \propto(d \phi / d a)^{-1} \propto$ $\left(\delta_{a} t_{\mathrm{arr}}\right)^{-1} \propto a_{\star}^{1 / 2}$ (See also Section 3.4.2).

Figure 11 shows the constraints on spin for pulsars similar to Ea, but having various $e_{\star}$. We can see that $\Delta a=0.03-0.004, \Delta i=7.0-1.0^{\circ}, \Delta \epsilon=10.0-2.0^{\circ}$. (or $\boldsymbol{\Delta} a=0.02-0.003, \boldsymbol{\Delta} i=4.0-0.8^{\circ}, \boldsymbol{\Delta} \epsilon=7.0-1.0^{\circ}$ ) if only the timing signals are used (or if both the timing and the position observations are used), when $e_{\star}$ varies from 0.3 to 0.9 .

These results suggest that pulsars with distance $<$ $600 \mathrm{AU}$ and $e_{\star} \gtrsim 0.3$ can set very tight constraint on the spin in three orbits. For these pulsars, note that the constraints on spin by additionally using the astrometric data are only modestly improved. This is because the constraints on spin parameters are dominated by the parts from the TOA, i.e., $\chi_{\mathrm{T}} \gg \chi_{\mathrm{P}}$.

\subsection{Constraints on the mass, distance and the proper motion of the $\mathrm{MBH}$}

Using both the astrometric measurements and the TOAs of pulsars in the MCMC simulations can provide precise estimate of the mass, distance and the proper motion of the MBH. The constraints on proper motion of the $\mathrm{MBH}$ for Ea are shown in Figure 12. We can see that the position of the $\mathrm{MBH}$ can be constrained to accuracies of $\boldsymbol{\Delta} X_{0}=8.1 \mu \mathrm{as}, \boldsymbol{\Delta} Y_{0}=8.0 \mu \mathrm{as}, \boldsymbol{\Delta} V_{x}=1.7 \mu \mathrm{as} / \mathrm{yr}$, $\Delta V_{y}=1.6 \mu \mathrm{as} / \mathrm{yr}$. The constraints on the mass and the $\mathrm{GC}$ distance are $\Delta M=10.6 M_{\odot}$ and $\Delta R_{\mathrm{GC}}=1.2 \mathrm{pc}$. Figure 13 shows constraints on the mass and the GC distance for pulsars in different orbital semimajor axes and eccentricities. From Figure 13 we can see that the mass can be constrained by a factor of $\sim 10^{-5}-10^{-6}$, i.e., $\Delta M_{\bullet}=1 \sim 10^{2} M_{\odot}$. The GC distance is constrained to an accuracy of $\sim 10^{-4}-10^{-3}$, i.e., $\Delta R_{\mathrm{GC}}=0.5 \sim 5 \mathrm{pc}$. Interestingly, we find that the constraints on $\mathrm{MBH}$ mass and GC distance are more accurate for pulsars at larger distances from the $\mathrm{MBH}$. The main reason is that relative position (or timing) error is inversely proportional to the semimajor axis of the star: Approximately, $\phi \propto \Delta_{\mathrm{R}} \propto a_{\star}$ and $|\mathbf{R}| \propto a_{\star}$, thus according to Equation 32 we have $\Delta M_{\bullet} \propto a_{\star}^{-1}, \Delta R_{\mathrm{GC}} \propto a_{\star}^{-1}$. These scaling relations are well consistent with those obtained by the MCMC simulations (See Figure 13).

Pulsars with high orbital eccentricities can help to put tighter constraints on the MBH mass, however, that does not appear to help in constraining the GC distance. 

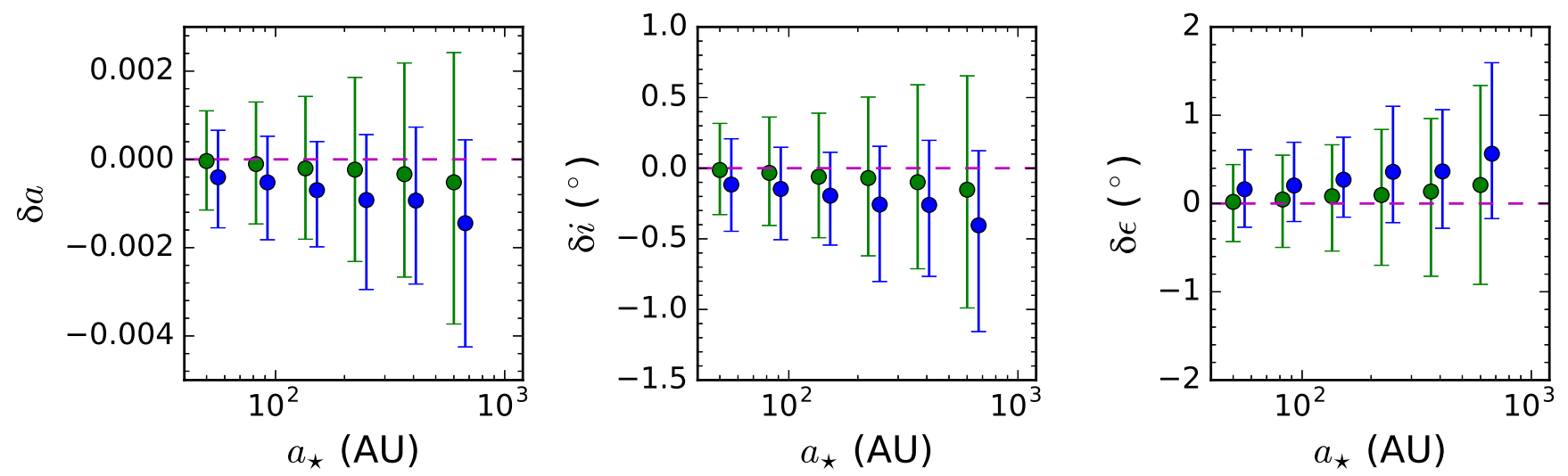

Figure 10. Constraints on the spin and its orientation as a function of the orbital semimajor axis of the pulsar. Here the pulsars are in orbits similar to Ea but the orbital semimajor axis varies from $50 \mathrm{AU}$ to $600 \mathrm{AU}$. The assumed measurement errors are $\sigma_{\mathrm{T}}=5 \mathrm{~ms}$ and $\sigma_{\mathrm{p}}=10 \mu \mathrm{as}$. The green dots show the MCMC results when only the TOAs are used while the blue dots are results when both the TOAs and the proper motion are used. Small offsets in $x$-axis are used for the blue dots for viewing clarity.
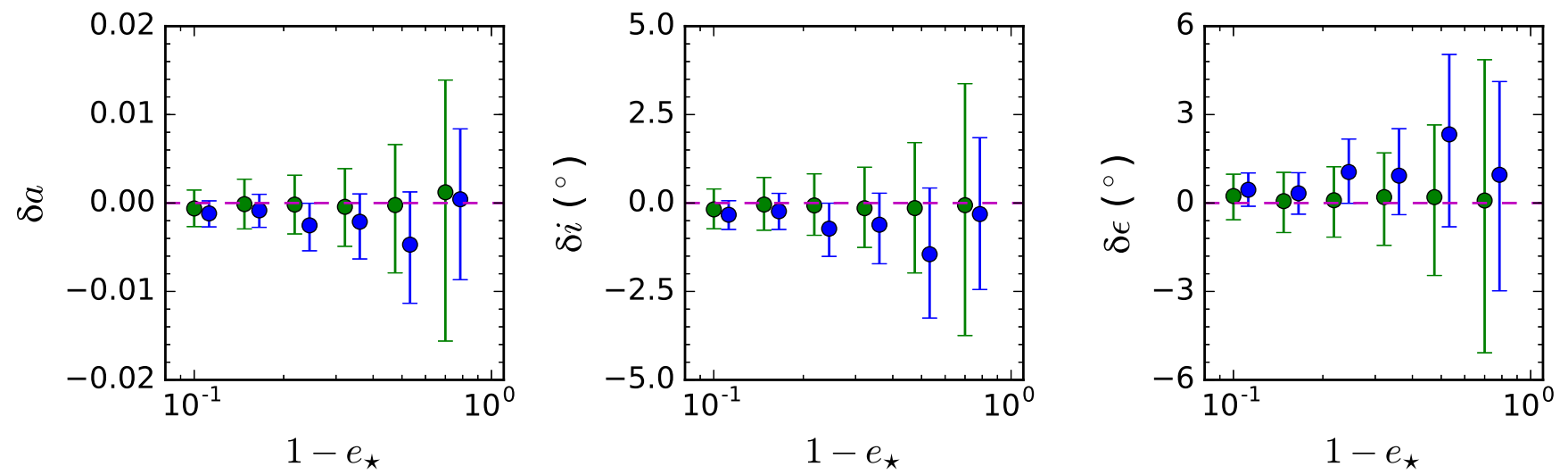

Figure 11. Similar to Figure 10 but with the eccentricity of the pulsars varying from 0.3 to 0.9 .
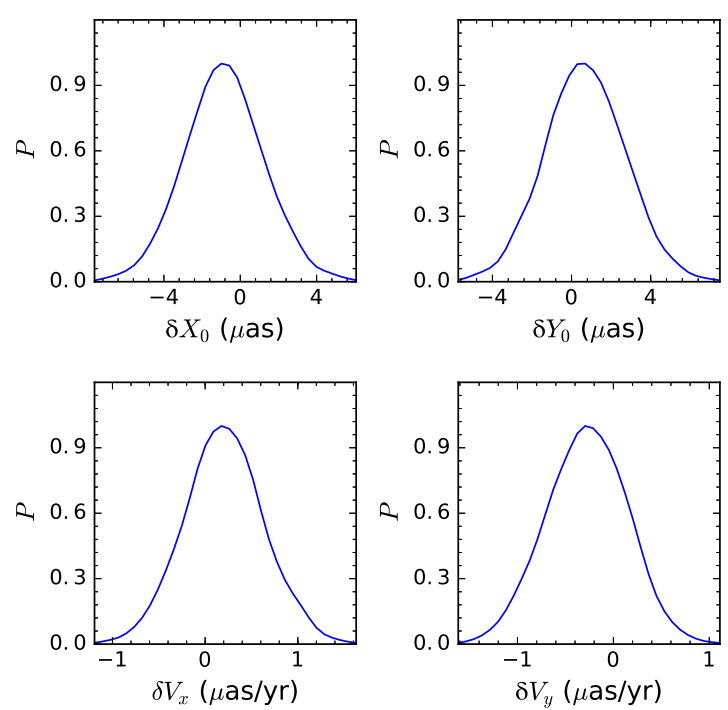

Figure 12. Constraints on the position and proper motion of the Sgr $A^{*}$ by observing both the timing and the apparent motion of the pulsar $\mathrm{Ea}$ in $8 \mathrm{yr}$. The assumed measurement errors are $\sigma_{\mathrm{T}}=5 \mathrm{~ms}$ and $\sigma_{\mathrm{p}}=10 \mu \mathrm{as}$.

\subsection{The effects of the pulsar's mass}

In our Kerr metric framework, the pulsar is a test particle and thus its mass is ignored. The difference in the pulsar timing due to this approximation, if there are any, should be of the order of the mass ratio, i.e., $10^{-7}-10^{-6}$ as the pulsar's mass is $m \sim 1.4 M_{\odot}$. However, considering that the timing accuracy of the pulsar is quite high, such differences could be detectable. Nevertheless, we find that the simulation results and conclusions in this work are only slightly affected by the pulsar's mass. The details of the estimations and the discussions are as follows.

In Newtonian physics, the orbital period of the pulsar is determined by the total mass of the binary. Thus, we expect that the measured mass of the $\mathrm{MBH}$ from our MCMC simulations should be effectively the sum of the true mass of the $\mathrm{MBH}$ and the pulsar. As the accuracy of MBH's mass obtained by GC pulsars is $\simeq 4-10^{2} M_{\odot}$ (See top left panel of Figure 13), the bias of the estimated MBH's mass should not be detectable, unless the pulsar is relatively far away ( $>1000 \mathrm{AU})$.

Both the Roemer delay and the Einstein delay are affected by the mass of the pulsar. Note that the Shapiro delay does not, as it depends only on the mass of the 

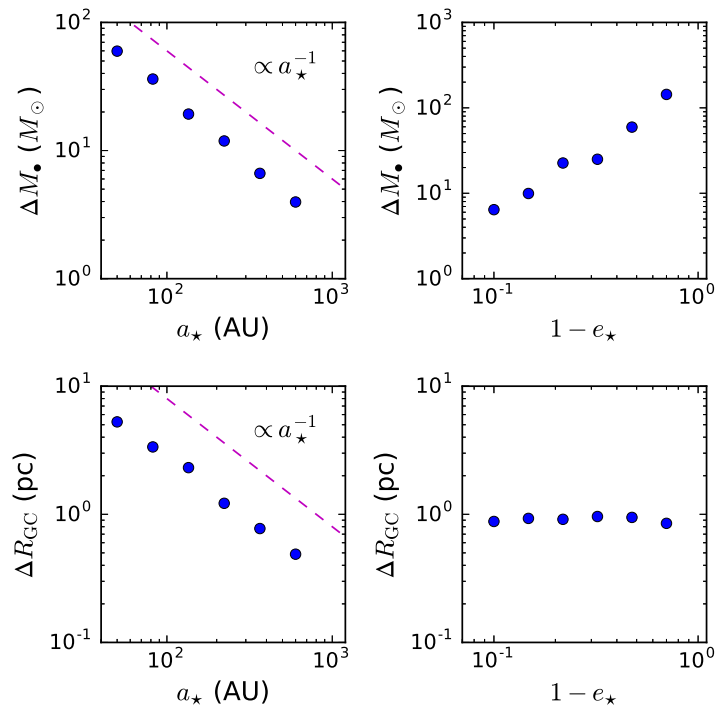

Figure 13. Constraints on the $\mathrm{MBH}$ mass $\left(\boldsymbol{\Delta} M_{\bullet}\right.$, upper panels) and GC distance $\left(\Delta R_{\mathrm{GC}}\right.$, lower panels) when both the timing and the proper motions are used, as functions of $a_{\star}$ (left panels) or $e_{\star}$ (right panels) of the pulsar. The other initial conditions of the pulsars are the same as Ea. The assumed measurement errors are $\sigma_{\mathrm{T}}=5 \mathrm{~ms}$ and $\sigma_{\mathrm{p}}=10 \mu \mathrm{as}$. The magenta lines in the top and bottom left panel show the reference scaling relations of $\propto a_{\star}^{-1}$.

MBH. Approximately, we have $\Delta_{R} \propto 1-m / M_{\bullet}$ and $\tilde{\gamma} \propto 1-\frac{1}{2} m / M_{\bullet}$ (Damour \& Deruelle 1986), where $m$ is the mass of the pulsar. For GC pulsars, we have $\Delta_{R}=10^{5}-10^{6} \mathrm{~s}$ and $\tilde{\gamma}=10^{4}-10^{5} \mathrm{~s}$ (See Figure 3), thus, the difference due to ignoring the pulsar mass should be $0.01-1 \mathrm{~s}$ and $1-100 \mathrm{~ms}$ for the Roemer delay and the Einstein delay, respectively. These differences may lead to biases on the parameters of the pulsar- $\mathrm{MBH}$ binary estimated by the MCMC simulations. Considering that the spin-induced effects are in orders of $10-100 \mathrm{~s}$ (See Figure 4or 5, top left panel), we expect that the spin parameters, if they are affected, should not be significantly biased from their true values.

The relativistic effects in the timing of pulsars are originated from the orbital precessions, which are also affected by the pulsar's mass. For example, The Schwarszchild orbital precession is proportional to $1+$ $m / M_{\bullet}$ and spin-induced orbital precessions are proportional to $1+\frac{7}{4} m / M \bullet$ (Wex \& Kopeikin 1999). Thus, the bias of these effects should be of the order of the mass ratio, i.e., $10^{-6}-10^{-7}$, which can be ignored.

We note that the effect of the pulsar's mass can be included by introducing a corresponding perturbative term into the Hamiltonian. However, deriving the explicit form of this term and such extensions of the current numerical method are beyond the scope of this work. We defer them to future studies.

\section{DISCUSSION}

Our results suggest that the spinning magnitude of the $\mathrm{MBH}$ can be constrained down to $\sim 10^{-2}$ within a decade even if the timing accuracies are relatively low, i.e., $\sigma_{\mathrm{T}} \sim 5 \mathrm{~ms}$. This suggests that SKA1-MID, not necessarily the final stage of SKA2, can already probe the spinning nature of the $\mathrm{GC} \mathrm{MBH}$, if any pulsar within $\lesssim 1000$ AU can be found. Indeed, it is suggested that
SKA1-MID is probably able to reveal the hidden pulsars at as low as $2.4 \mathrm{GHz}$ with spin period $\sim 0.5 \mathrm{~s}$ in this region (Eatough et al. 2015).

Our simulations have the advantage that all quantities are obtained under the Kerr metric. The signals could be more accurate than those of the previous studies that are based on post-Newtonian approximation methods. The main disadvantages of our method are that the gravitational wave decay is not included, and that the torque effects are not easy to be discussed separately. As a consequence, alternative-gravity theories are hard to discuss in a theory-independent way. Anyway, the deviations of the GR can still be detected by comparing our model predictions with the observables, if the full GR model could not fit the observations well, i.e., if the MCMC parametric fits leave significant residuals.

Note that some GR effects unique to pulsar timing are not covered in this work. For example, the shift of the time of emission of the pulse centroid due to the spinning precession of the pulsar, the distortion of the pulse profile (e.g., Rafikov \& Lai 2006), or the high order pulses due to extremely strong gravitational bending (Wang et al. 2009). These effects can be straightforwardly included in our framework as the geodesic equation and the light trajectories have been solved explicitly in our method. However we notice that some of these effects are significant only if the pulsars are in edge-on orbit (e.g., Rafikov \& Lai 2006), thus they have negligible effects for results shown in this study.

The pulsars could be perturbed by other surrounding gravitational sources, e.g., the pulsars or other stellar remnants. The effects of the background perturbation are expected to be important outside $\gtrsim 100-$ 400 AU (Merritt et al. 2010; Zhang \& Iorio 2017). Due to the different nature of these background perturbations, they are expected to be separable from the GR effects (Angélil \& Saha 2014; Zhang \& Iorio 2017).

\section{CONCLUSIONS}

It is believed that pulsars rotating closely around the $\mathrm{GC} \mathrm{MBH}$ are superb tools in probing the GR and the gravity theories. Based on a relativistic framework developed in our previous work, here we study both the TOA and the apparent motion of these pulsars and the corresponding spin-induced effects. We take the pulsar as a test particle and solve explicitly the geodesic equations of the pulsar's motion and its pulse trajectories to the observer in the Kerr metric. By performing a number of MCMC simulations, we investigate the constraints on the spin and other properties of the $\mathrm{GC} \mathrm{MBH}$ achievable by monitoring surrounding pulsars.

We find that the full GR treatment is necessary in describing accurately the timing signals. If approximate models are used, that assume that the orbital precession increases linearly with time, the predicted TOA difference due to spin effects can deviate from the results of our relativistic simulations up to $\sim 10 \mathrm{~s}$, which would be quite apparent for timing observations performed by future facilities, e.g., the SKA.

We find that the spin-induced TOA differences can mount up to $\sim 140 \mathrm{~s}$ in $\sim 8$ yr for a pulsar with orbital period of $\sim 2.6$ yr. Even for S2-like or S0-102-like pulsars, the spin-induced TOA differences can be up to $\sim 80 \mathrm{~s}$ $(\sim 20 \mathrm{~s})$ after $40 \mathrm{yr}(30 \mathrm{yr})$ of observation. The signal 
is orders of magnitude larger than the timing accuracies expected in the future $(\lesssim 1-10 \mathrm{~ms})$, thus it should be possible to set tight constraints on the spinning of the $\mathrm{MBH}$.

We perform a number of MCMC simulations to study the constraints on the spinning of the $\mathrm{MBH}$. We find that strong near-degeneracies among the spin parameters could appear, if only the timing of pulses are used. Such near degeneracies can be weakened if the pulsar proper motion is measured with considerable accuracies along with the timing, or if the pulsar is close enough to the $\mathrm{MBH}$ such that the orbital precession or the quadrupolemoment effects are significant.

Although near degeneracies exist if only the timing of pulsars are used, the constraints of the spinning parameters are still very tight. By monitoring a normal pulsar with orbital period of $\sim 2.6 \mathrm{yr}$ and eccentricity of $0.3-0.9$, and assuming the timing accuracy of $1-5 \mathrm{~ms}$, we find that within $\sim 8 \mathrm{yr}$ the magnitude, the line of sight inclination and the position angle of the $\mathrm{MBH}$ spin can be constrained with $2 \sigma$ error given by $10^{-3}-10^{-2}$ and $10^{-1}-5^{\circ}, 10^{-1}-10^{\circ}$, respectively.

Even for pulsars in orbits similar to the currently detected star S2/S0-2 or S0-102 and providing that the timing accuracy is $\sim 5 \mathrm{~ms}$, we find that the spinning of the MBH can still be constrained within $4-8 \mathrm{yr}$. The most significant constraints of the spin parameters are provided near pericenter passage. Thus, in the optimistic case that the timing observations start near the pericenter passages of pulsars, the spinning of the $\mathrm{MBH}$ can be constrained within $2-4$ yr.

If the proper motion of the pulsars with accuracy of $10 \mu$ as can also be collected along with the timing measurement, then the position, velocity, mass and the distance of the $\mathrm{MBH}$ can be constrained about $\sim 10 \mu \mathrm{as}$, $\sim 10 \mu \mathrm{as} / \mathrm{yr}, \sim 1 M_{\odot}$ and $\sim 1 \mathrm{pc}$, respectively.

We are very grateful to the anonymous referee for all the suggestions have given to us, which have improved this paper significantly. We thank Zhu Weishan for providing the computing resource to the TianHe II National Supercomputer Center in Guangzhou, on which part of the simulations are performed. This work was supported in part by the National Natural Science Foundation of China under grant No. 11603083, 11673077. This work was also supported in part by "the Fundamental Research Funds for the Central Universities" grant No. 161GPY51, the Key Project of the National Natural Science Foundation of China under grant No. 11733010. Part of the numerical work was performed in the computing cluster in School of Physics and Astronomy, Sun Yat-Sen University.

\section{REFERENCES}

Angélil, R., Saha, P., \& Merritt, D. 2010, ApJ, 720, 1303

Angélil, R., \& Saha, P. 2010, ApJ, 711, 157

Angélil, R., \& Saha, P. 2011, ApJL, 734, 19

Angélil, R., \& Saha, P. 2014, MNRAS, 444, 3780
Bates, S. D., Johnston, S., Lorimer, D. R., et al. 2011, MNRAS, 411,1575

Blandford, R., \& Teukolsky, S. A. 1976, ApJ, 205, 580

Cordes, J. M., \& Lazio, T. J. W. 1997, ApJ, 475, 557

Chennamangalam, J., \& Lorimer, D. R. 2014, MNRAS, 440, L86

Damour, T., \& Deruelle, N. 1986, Ann. Inst. Henri Poincaré

Phys. Théor., Vol. 44, No. 3, p. 263 - 292, 44, 263

Deneva, J. S., Cordes, J. M., \& Lazio, T. J. W. 2009, ApJ, 702, L177

Eatough, R. P., Falcke, H., Karuppusamy, R., et al. 2013, Nature, 501,391

Eatough, R., Lazio, T. J. W., Casanellas, J., et al. 2015, Advancing Astrophysics with the Square Kilometre Array (AASKA14), 45

Edwards, R. T., Hobbs, G. B., \& Manchester, R. N. 2006, MNRAS, 372, 1549

Eisenhauer, F., Genzel, R., Alexander, T., et al. 2005, ApJ, 628, 246

Fomalont, E., \& Reid, M. 2004, New Astronomy Review, 48, 1473

Ghez, A., Salim, S., Weinberg, N. N., Lu, J. R., Do, T., Dunn, J. K., Matthews, K., Morris, M. R., Yelda, S., Becklin, E. E., et al. 2008, ApJ, 689, 1044

Gillessen, S., Eisenhauer, F., Trippe, S., Alexander, T., Genzel, R., Martins, F., \& Ott, T. 2009, ApJ, 692, 1075

Gillessen, S., Plewa, P. M., Eisenhauer, F., et al. 2017, ApJ, 837, 30

Godfrey, L. E. H., Bignall, H., Tingay, S., et al. 2012,

Publications of the Astronomical Society of Australia, 29, 42

Hobbs, G. B., Edwards, R. T., \& Manchester, R. N. 2006 , MNRAS, 369, 655

Jaroszynski, M. 1998, Acta Astron., 48, 653

Kopeikin, S. M. 1996, ApJ, 467, L93

Kramer, M. et al., 2006, Science, 314, 97

Lense, J., \& Thirring, H. 1918, Physikalische Zeitschrift, 19,

Liu, K., Wex, N., Kramer, M., Cordes, J. M., \& Lazio, T. J. W. 2012, ApJ, 747, 1

Lorimer, D. R. 2008, Living Reviews in Relativity, 11,

Lu, J. R., Do, T., Ghez, A. M., et al. 2013, ApJ, 764, 155

Macquart, J.-P., Kanekar, N., Frail, D. A., \& Ransom, S. M. 2010, ApJ, 715, 939

Merritt, D., Alexander, T., Mikkola, S., \& Will, C. M. 2010,

Phys. Rev. D, 81, 062002

Merritt, D., Alexander, T., Mikkola, S., \& Will, C. M. 2011, Phys. Rev. D, 84, 044024

Meyer, L., Ghez, A. M., Schödel, R., et al. 2012, Science, 338, 84 Rafikov, R. R., \& Lai, D. 2006, ApJ, 641, 438

Rea, N., Esposito, P., Pons, J. A., et al. 2013, ApJ, 775, L34

Reid, M. J., \& Brunthaler, A. 2004, ApJ, 616, 872

Rubilar, G. F., \& Eckart, A. 2001, A\&A, 374, 95

Paumard, T., Genzel, R., Martins, F., et al. 2006, ApJ, 643, 1011

Peters, P. C. 1964, Phy. Rev., 136, 1224

Pfahl, E., \& Loeb, A. 2004, ApJ, 615, 253

Psaltis, D., Wex, N., \& Kramer, M. 2016, ApJ, 818, 121

Shao, L., Stairs, I., Antoniadis, J., et al. 2015, Advancing Astrophysics with the Square Kilometre Array (AASKA14), 42 Shapiro, I. I. 1964, Physical Review Letters, 13, 789

Stairs, I. H. 2003, Living Reviews in Relativity, 6, 5

Taylor, J. H., \& Weisberg, J. M. 1989, ApJ, 345, 434

Taylor, J. H., 1994, Rev. Mod. Phys., 66, 711

Yu, Q., Zhang, F., \& Lu, Y. 2016, ApJ, 827, 114

Wang, Y., Jenet, F. A., Creighton, T., \& Price, R. H. 2009, ApJ, 697, 237

Wex, N. 1995, Classical and Quantum Gravity, 12, 983

Wex, N., \& Kopeikin, S. M. 1999, ApJ, 514, 388

Will, C. M. 2008, ApJ, 674, L25

Will, C. M. 2014, Living Reviews in Relativity, 17, 4

Zhang, F., Lu, Y., \& Yu, Q. 2014, ApJ, 784, 106

Zhang, F., Lu, Y., \& Yu, Q. 2015, ApJ, 809, 127

Zhang, F., \& Iorio, L. 2017, ApJ, 834, 198 